D) Check for updates

Cite this: Org. Chem. Front., 2020, 7 3001

Received 28th May 2020,

Accepted 21st July 2020

DOI: 10.1039/d0qo00637h

rsc.li/frontiers-organic

\section{Recent progress in the usage of tetrabromo- substituted naphthalenetetracarboxylic dianhydride as a building block to construct organic semiconductors and their applications}

\begin{abstract}
Cheng Zhang, ${ }^{\mathrm{a}, \mathrm{b}}$ Zongrui Wang, ${ }^{\mathrm{b}}$ Hua Li, ${ }^{\text {a }}$ Jianmei Lu (D)*a and Qichun Zhang (D) *b,c
Tetrabromo-substituted naphthalenetetracarboxylic dianhydrides (TBNDA) have attracted considerable attention in constructing organic semiconductors (OSCs) for diverse applications in the past decade. A series of synthetic strategies have been developed to modify the parent structure, including longitudinal imidization and lateral core-functionalization. The as-obtained small molecular and polymeric materials present expanded conjugated systems with unique characteristics of near-infrared absorption, outstanding $\pi$-acidity, and low-lying LUMO levels. In this review, recent progress in the synthetic methods of the TBNDI derivatives and their applications in organic field-effect transistors (OFETs), gas sensors, fluoride sensors, and supramolecular self-assembly are summarized. The remaining opportunities and challenges are elaborated to give specific guidance on preparing abundant TBNDA derivatives and their anticipated applications.
\end{abstract}

\section{Introduction}

${ }^{a}$ College of Chemistry, Chemical Engineering and Materials Science, Collaborative Innovation Center of Suzhou Nano Science and Technology, Soochow University, Suzhou 215123, P. R. China.E-mail: lihuaw@suda.edu.cn, lujm@suzqda.edu.cn ${ }^{b}$ School of Materials Science and Engineering, Nanyang Technological University, Singapore 639798, Singapore.E-mail: qczhang@ntu.edu.sg

${ }^{c}$ Department of Materials Science and Engineering, City University of Hong Kong, Kowloon, Hong Kong, China. E-mail: qiczhang@cityu.edu.hk
Organic semiconducting materials (OSCs), including n-type, p-type, and ambipolar, have been extensively investigated over the past three decades for their intrinsic properties and a variety of applications in organic optoelectronics such as photovoltaic cells, solar cells, field-effect transistors, energy storage devices, memory devices, and so on. ${ }^{1-9}$ Among them,

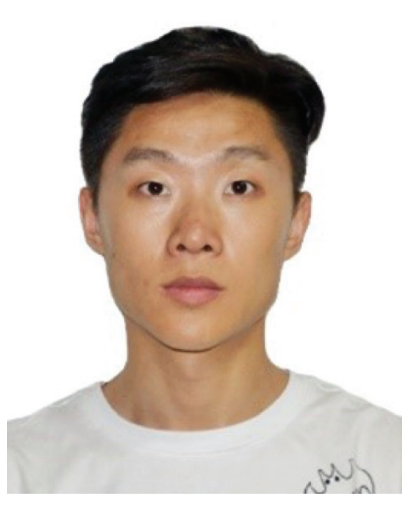

Cheng Zhang
Cheng Zhang is currently a PhD student under the supervision of Prof. Jianmei Lu at the College of Chemistry, Chemical Engineering and Materials Science, Soochow University. In 2019, he joined Prof. Qichun Zhang's group at the School of Materials Science and Engineering, Nanyang Technological University, as a joint $\mathrm{PhD}$ student. His research interests include the synthesis and application of organic semiconductor materials, particularly organic resistive memory devices.

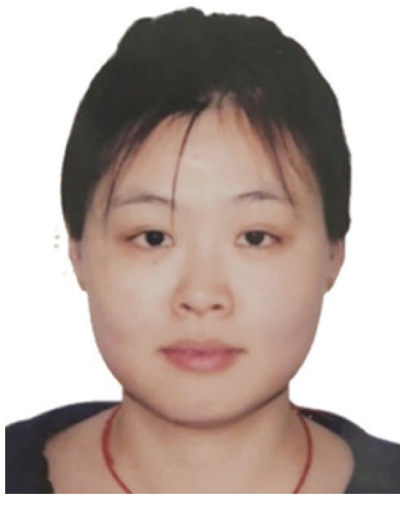

Zongrui Wang
Zongrui Wang received her B.S. degree in Chemistry in 2010 from the School of Chemistry and Chemical Engineering at Inner Mongolia University, and then received her Ph.D. from the Institute of Chemistry, Chinese Academy of Science in July 2016 under the co-supervision of Profs Huanli Dong and Wenping Hu. After that, she joined the group of Associate Professor Qichun Zhang in Nanyang Technological University as a Research Fellow. Her present research focuses on organic electronics including the design, assemble technology and optoelectronic properties of organic cocrystals. 
naphthalene tetracarboxylic dianhydride (NDA) and its derivatives have been demonstrated as one of the most promising electron-deficient building blocks to construct air-stable n-type OSCs. ${ }^{10-13}$ NDA moieties, with the features of a coplanar conjugated structure, high electron affinity, and thermal and photochemical stability, have been diversely functionalized through attaching different groups on the nitrogen atoms of diimide or the aromatic core. Such modifications could create various high-performance solution-processable materials with lowlying highest occupied molecular orbitals (HOMO) and lowest unoccupied molecular orbitals (LUMO) as well as high crystallinity.

The high-yield synthesis of 2,3,6,7-tetrabromonaphthalene dianhydride (TBNDA) and 2,3,6,7-tetrabromonaphthalene diimide (TBNDI) was firstly reported through the bromination and imidization of NDA by Zhu et al. and Würthner et al., which successfully opened a new door for the construction of tetrasubstituted NDA derivatives. ${ }^{14,15}$ As a promising building block for expanding the $\pi$-conjugation of the NDA core, TBNDA has attracted much attention due to the possibility of realizing superior optical and electrical properties through the core functionalization. Compared to 2,6-dichloronaphthalene dianhydride (DCNDA) or 2-bromonathalene dianhydride (MBNDA) and 2,6-dibromonaphthalene dianhydride (DBNDA), TBNDA exhibits a greater advantage in the core-expansion with heterocyclic rings (containing heterocyclic atoms, i.e., $\mathrm{O}, \mathrm{S}, \mathrm{N}$ ) through a nucleophilic halogen-exchange reaction, nucleophilic substitution, and a metal-catalyzed Suzuki or Stille crosscoupling reaction, where the unfavorable polymerization could be excluded due to the detrimental multi-reaction sites. ${ }^{16-19}$

Starting with NDA, the building unit TBNDA could be prepared as a yellow but highly insoluble compound, which was directly used for the next step without further purification. As shown in Scheme 1, three strategies have been explored to produce different TBNDA derivatives over the years. In strategy I, the esterification of dianhydride can improve solution processability, which ensures that the hydrolyzation can efficiently introduce a series of functional monomeric units through the replacement of the $\mathrm{Br}$ group, although the reactivity of four $\mathrm{Br}$ atoms is somewhat decreased. Strategy II with the direct functionalization of the TBNDA core is rarely reported due to its extremely poor solubility. Strategy III has been widely utilized in many reports, where the longitudinal imidization of TBNDA was firstly performed on $\mathrm{N}$ atoms with different side chains, such as linear or branched alkyl chains, semifluoroalkyl side chains, and hybrid siloxane chains. These asobtained TBNDI intermediates would act as new building blocks to construct a series of organic $\pi$-conjugated semiconducting materials. In the context of TBNDI-based materials (TBNDIs), the reported designs of molecular structures can be divided into three types: (a) flanking annulations (i.e., unilateral and bilateral annulation, symmetric and asymmetric annulation) are selected to extend the $\pi$-system with heteroaromatic rings (containing $\mathrm{N}, \mathrm{S}, \mathrm{O}$ ) or carbocyclic rings; (b) the flanking $\mathrm{Br}$ atoms are replaced by non-annulation moieties such as cyano, amino, alkyl and aryl groups; and, (c) extension of $\pi$-conjugated dimeric TBNDI (bis-TBNDI) precursors and analogues (bis-TBNDIs) could be realized through a nucleophilic or $\mathrm{C}-\mathrm{C}$ coupling reaction.

In this review, we summarize recent strategies for the structural extensions of TBNDA and the physical properties of asobtained materials. Moreover, diverse applications are also presented, including organic field-effect transistors (OFETs), fluoride sensors with high selectivity and sensitivity, supramolecular self-assembly and polymorphic transitions, OFETbased gas sensors and thermoelectric devices, stable radical anions, self-colored nanoparticles for DNA probes and NIR J-aggregation behaviors. Given that the exploration of TBNDA

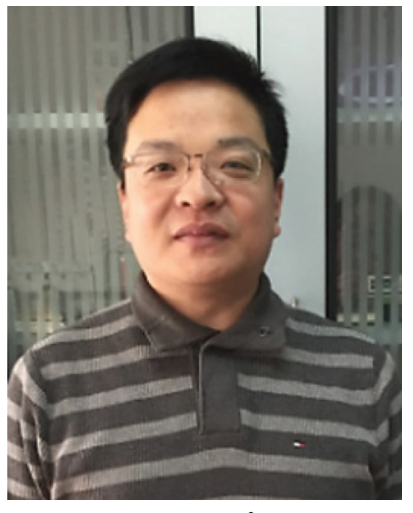

Hua Li ance organic semiconductor materials and organic resistive memory devices.
Hua Li received his $B S$ and $P h D$ degrees in applied chemistry at the College of Chemistry, Chemical Engineering and Materials Science, Soochow University, under the supervision of Prof. Jianmei Lu in 2000 and 2011, respectively. He is currently a professor at the College of Chemistry, Chemical Engineering and Materials Science, Soochow University. His research focuses on the design and preparation of high-perform-

\section{tor}

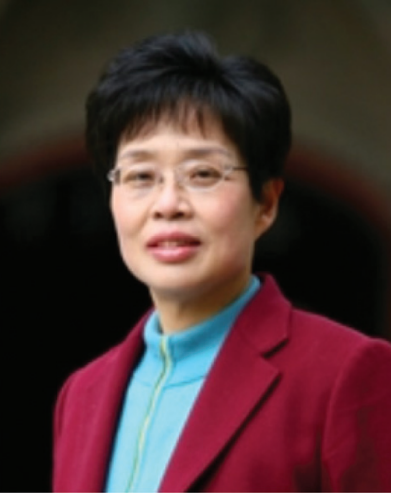

Jianmei Lu
Jianmei Lu received her $P h D$ degree in polymer chemistry from Zhejiang University in China and was then appointed as a full professor at the College of Chemistry, Chemical Engineering and Materials Science, Soochow University in 2000. She is currently a fellow of the Chemical Industry and Engineering Society of China and the vice president of Soochow University. She has particular research interests in the development of materials for nonvolatile electronic memory devices, organic sensors, smart electronics, adsorption of oils, and treatment. 


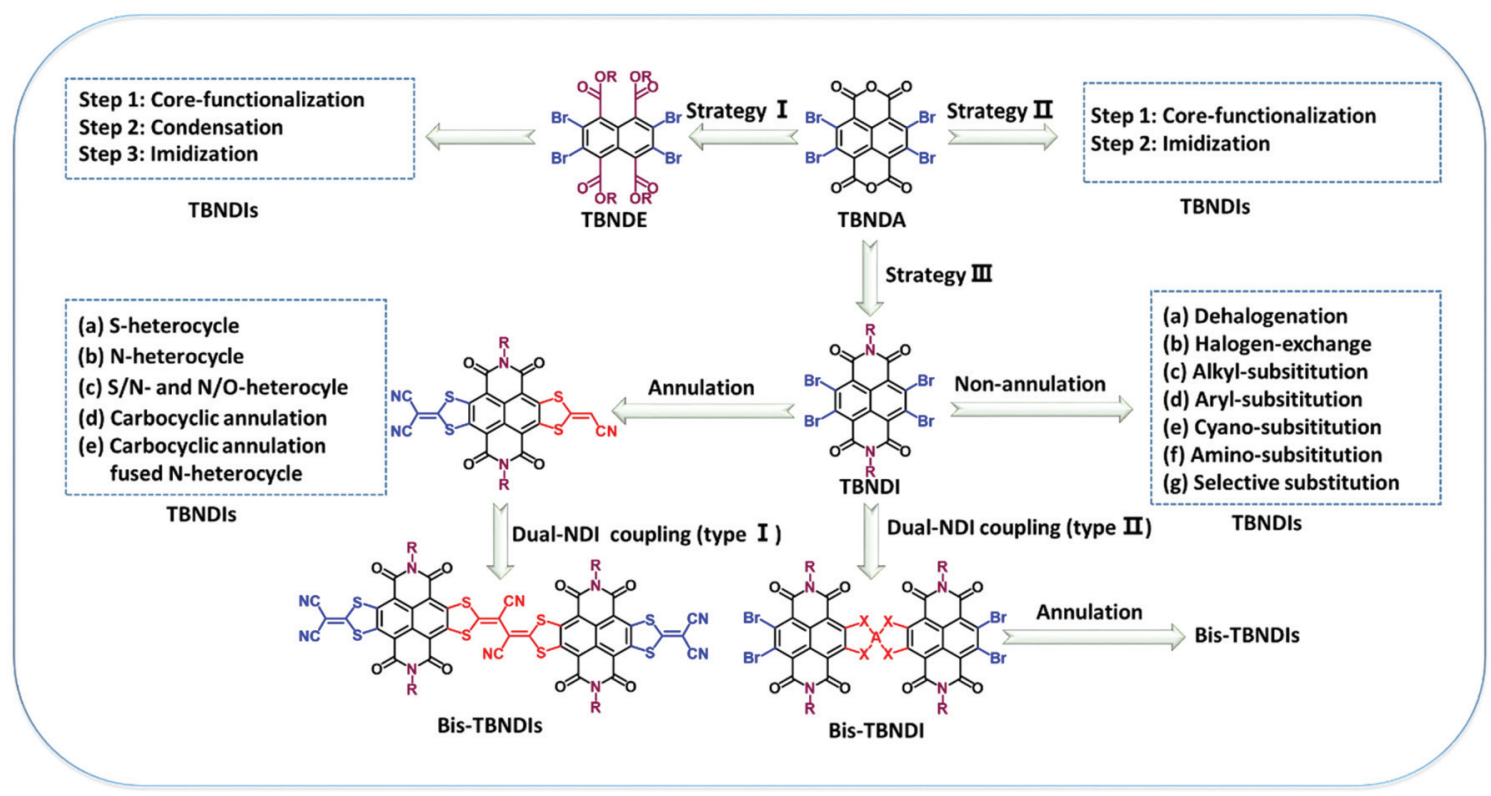

Scheme 1 Commonly-used synthetic strategies with TBNDA and TBNDI derivatives as building blocks.

is a relatively young area, we believe that this review will provide some guidelines in exploiting more promising TBNDAbased materials with superior properties in the future.

\section{Synthesis and molecular design}

\subsection{Synthesis of TBNDA precursor}

Although TBNDA was observed in the reaction between NDA and bromine $\left(\mathrm{Br}_{2}\right)$ in $\mathrm{H}_{2} \mathrm{SO}_{4}$ in 1981, it was always considered as a byproduct. ${ }^{14,15}$ Until 2006, high-purity TBNDA was firstly obtained in a large amount by brominating NDA with elemen-

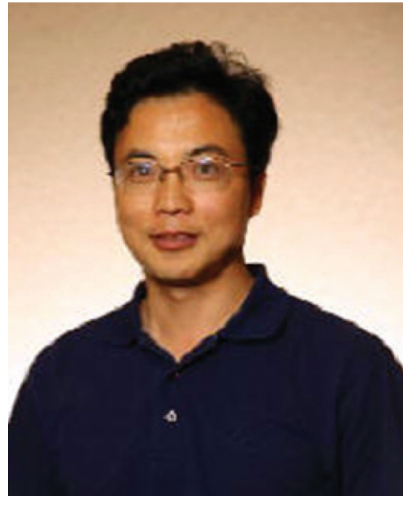

Qichun Zhang
Qichun Zhang is an associate professor at the School of Materials Science and Engineering at Nanyang Technological University, Singapore. In 2014, he was promoted to associate professor with tenure. His research interests include carbon-rich conjugated materials and their applications (novel organic conjugated materials for optoelectronic and semiconductor devices, novel electrode materials, and new design for microbial fuel cells). He is currently a Fellow of the Royal Society of Chemistry. In 2018 and 2019, he has been recognized as one of the highly-cited researchers (top $1 \%)$ in the cross-field in Clarivate Analytics. Till now, he has published more than 377 papers and $H$ index: 75. tal bromine $\left(\mathrm{Br}_{2}\right)$ in a mixture of $98 \mathrm{wt} \% \mathrm{H}_{2} \mathrm{SO}_{4}$ and oleum at $140{ }^{\circ} \mathrm{C}$ for 4 weeks. ${ }^{14}$ Piyakulawat et al. duplicated this reaction with oleum $\left(65 \% \mathrm{SO}_{3}\right)$ at a mild room temperature (r.t.). ${ }^{20}$ Würthner et al. reported a more efficient bromination in oleum $\left(20 \% \mathrm{SO}_{3}\right)$ at r.t. in the presence of dibromoisocyanuric acid (DBI). This methodology selectively produced TBNDA in high purity and yield, which was confirmed by ${ }^{1} \mathrm{H}$ NMR spectroscopy (DMSO- $d_{6}$ ) without any proton resonance, elemental analysis (EA) and EI mass spectrometry. ${ }^{15}$ The modified reaction conditions of the first oleum $\left(20 \% \mathrm{SO}_{3}\right)$ at $55{ }^{\circ} \mathrm{C}$ for $2 \mathrm{~h}$ and then at $85{ }^{\circ} \mathrm{C}$ for $50 \mathrm{~h}$ were applied, but afforded overequivalent products because of the unknown purity. ${ }^{21}$ Since the products are insoluble, the desired TBNDA was difficult to isolate or further purify. Thus, the actual yields of TBNDA could be much lower than the reported results. Subsequently, some other regioselective brominating reagents including 5,5dimethyl-1,3-dibromohydantoin (DBH), tribromoisocyanuric acid (TBCA), and sodium bromide (NaBr), and different reaction conditions were utilized to fulfill this reaction. ${ }^{22-24}$ All the bromination methods obtained from the literature are listed in Table 1, where Govindaraju et al. realized the synthesis of TBNDA (yield: $96 \%$ ) by stirring $98 \% \mathrm{H}_{2} \mathrm{SO}_{4}$ and 3 eq. of $\mathrm{DBH}$ at r.t. for $4 \mathrm{~h}$ and then at $80^{\circ} \mathrm{C}$ for $12 \mathrm{~h}^{22}$ For additional confirmation of the purity of TBNDA gained with DBH reagent, TBNDA was converted into the alkyl-substituted product via imidization with 3 -eq. (equivalent) of $n$-octylamine in glacial acetic acid (HOAc) at $130{ }^{\circ} \mathrm{C}$ for $6 \mathrm{~h}$. The majority of the obtained ring-opening tetrabromo-substituted product and a trace of core-substituted products demonstrated the reliability of the high yield and purity. ${ }^{22}$ For the TBCA method, it revealed a highly regioselective bromination to afford MBNDA, DBNDA and TBNDA by modifying the equivalents of TBCA and the reaction time. Under the reaction conditions of r.t. for $8 \mathrm{~h}$ 
Table 1 The summary of various synthetic conditions to produce TBNDA

\begin{tabular}{|c|c|c|c|c|}
\hline $\begin{array}{l}\text { Brominating } \\
\text { reagent }\end{array}$ & Solvent \& catalyst & $\begin{array}{l}\text { Reaction } \\
\text { conditions }\end{array}$ & Yield & Ref. \\
\hline $\mathrm{Br}_{2}$ (5.3 eq.) & $\begin{array}{l}98 \mathrm{wt} \% \mathrm{H}_{2} \mathrm{SO}_{4} \text { and } \\
\text { oleum }(\mathrm{V}: \mathrm{V}=4: 1) \\
\text { iodine }\end{array}$ & $\begin{array}{l}140^{\circ} \mathrm{C}, 4 \\
\text { weeks }\end{array}$ & $96 \%$ & 14 \\
\hline $\mathrm{Br}_{2}$ (2.1 eq.) & $\begin{array}{l}\text { Oleum }\left(65 \% \mathrm{SO}_{3}\right) \\
\text { iodine }\end{array}$ & R.t., $15 \mathrm{~h}$ & $95 \%$ & 20 \\
\hline DBI (2.5 eq.) & Oleum $\left(20 \% \mathrm{SO}_{3}\right)$ & R.t., $3 \mathrm{~h}$ & $93 \%$ & 15 \\
\hline DBI (2.0 eq.) & Oleum $\left(20 \% \mathrm{SO}_{3}\right)$ & $\begin{array}{l}55^{\circ} \mathrm{C}, 2 \mathrm{~h} ; \\
85^{\circ} \mathrm{C}, 50 \mathrm{~h}\end{array}$ & - & 21 \\
\hline DBH (3.0 eq.) & Conc. $\mathrm{H}_{2} \mathrm{SO}_{4}$ & $\begin{array}{l}\text { R.t., } 4 \mathrm{~h} ; \\
80^{\circ} \mathrm{C}, 12 \mathrm{~h}\end{array}$ & $96 \%$ & 22 \\
\hline TBCA (2.5 eq.) & Conc. $\mathrm{H}_{2} \mathrm{SO}_{4}$ & $\begin{array}{l}\text { R.t., } 8 \mathrm{~h} \text {; } \\
80^{\circ} \mathrm{C}, 8 \mathrm{~h}\end{array}$ & $67 \%$ & 23 \\
\hline $\mathrm{NaBr}$ (4.4 eq.) & Oleum $\left(20 \% \mathrm{SO}_{3}\right)$ & $180^{\circ} \mathrm{C}, 4 \mathrm{~h}$ & $62 \%$ & 24 \\
\hline
\end{tabular}

and then at $80{ }^{\circ} \mathrm{C}$ for $8 \mathrm{~h}$, a pale yellow crude product with $98 \%$ yield was obtained, followed by recrystallization in DMF to give the pure product as white crystals in $87 \%$ yield. ${ }^{23} \mathrm{NDA}$, 4.4-eq. $\mathrm{NaBr}$ and oleum $\left(20 \% \mathrm{SO}_{3}\right)$ were stirred in a Carrius tube at $180{ }^{\circ} \mathrm{C}$ for $4 \mathrm{~h}$, followed by slow addition of propionic acid (CAUTION: highly exothermic) to give desired TBNDA in $62 \%$ yield, which was characterized by mass spectrometry and the diagnostic isotopic pattern. ${ }^{24}$ Crystals of TBNDA were grown by the method of vapor diffusion from water vapor to DMSO solution. A twisted aromatic core was observed, which could be attributed to the steric effect of the four ortho bromines. ${ }^{24}$ To sum up, the advantages and disadvantages of these agents are apparent. For example, $\mathrm{Br}_{2}$, as a reagent in the presence of a catalytic amount of iodine, always brought several difficulties in the reaction and product treatment due to the hazardous and corrosive nature of unreacted $\mathrm{Br}_{2}$ and the byproduct $\mathrm{HBr}$. For $\mathrm{NaBr}$ as a reagent, a high temperature $\left(180^{\circ} \mathrm{C}\right)$ was required to carry out this reaction. Therefore, the brominating reagents DBI, DBH and TBCA were recommended as efficient, low-cost, regioselective and high atom-economy for the synthesis of MBNDA, DBNDA and TBNDA with the advantages of mild reaction conditions, short reaction times, and high conversions. Furthermore the recrystallization technique could be effectively utilized to isolate the desired product in high purity.

\subsection{Strategy I: esterification of dianhydride}

As shown in Scheme 2, esterification of dianhydride was employed to improve the solubility of TBNDA, as well as to enhance the molecular self-assembly and stacking. For example, TBNDA could be esterified with $n$-bromobutane $(n$-BuBr) or iodoethane (EtI) under alkaline conditions to produce a white compound TBNDE with a high yield. Subsequently, the Stille cross-coupling reaction between organotin reagents and tetra-ester TBNDE would be carried out more easily in toluene to afford compounds $\mathbf{1}$ and 2, where the yield could be increased with a prolonged reaction time. The tetra-alkyl substituted NDA intermediate (compound 3) was prepared successfully, which could be used as building blocks to construct large $\pi$-conjugated backbones at the longitudinal positions. ${ }^{25}$ Thus, the TBNDI derivative (4) and ladder-like $\pi$-conjugated molecules (5 and 6) could be obtained through the condensation reactions between 3 and the respective alkylamine and $o$-phenylenediamine. The ladder-like $\pi$-conjugated compounds 5 and 6 with a rigid two-dimensional planar ribbon-pattern framework were classified as poly(benzobisimidazobenzophen-anthroline) (BBL) analogues, which have attracted significant interest due to the excellent stability and exceptional $n$-channel behaviors. The success in synthesizing high soluble rigid compounds $\mathbf{5}$ and $\mathbf{6}$ also indicates that this strategy is an efficient method to address the issue of insolubility for the common rigid structures, which permits that more intriguing BBL-like polymers or small molecules with enhanced solubility will be developed in the near future. ${ }^{26-28}$ Compounds 7 with annulated four-membered rings could be prepared through attaching two triple bonds onto TBNDE to form compound 2, followed by the annulation with di(trimethylsilane)acytelyene in the presence of the catalyst $\mathrm{CpCo}$ $(\mathrm{CO})_{2}$ and then hydrolysis with trifluoroacetic acid (TFA) in

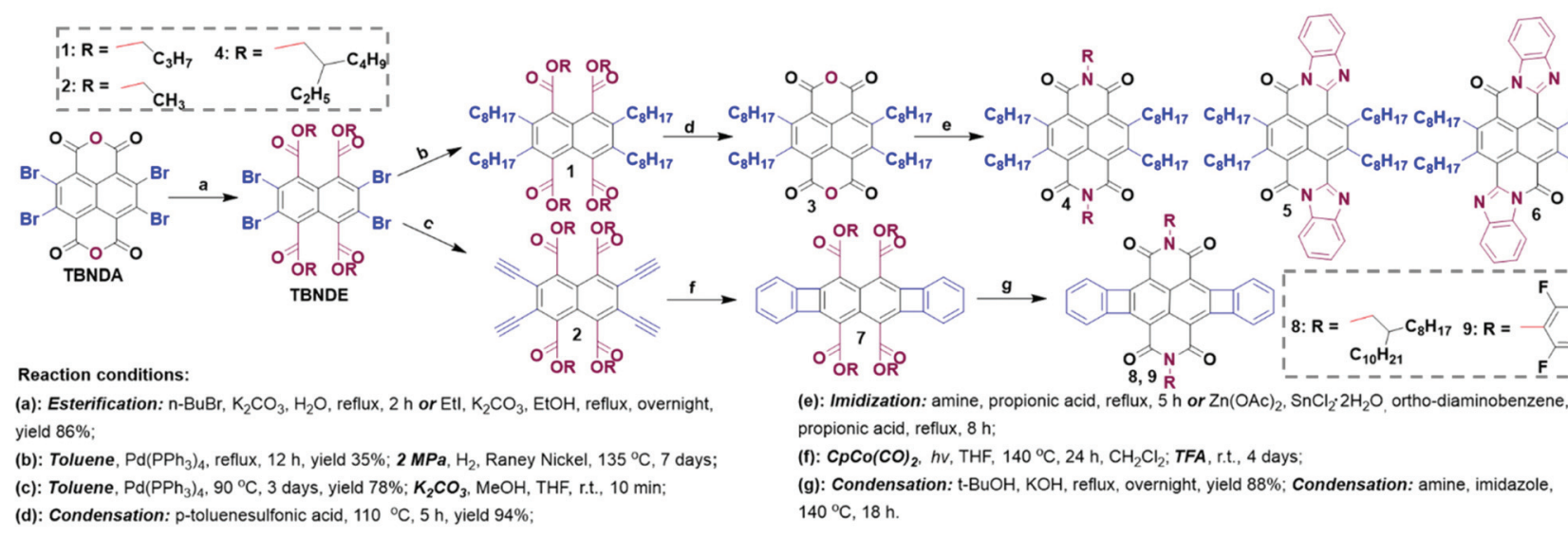

Scheme 2 Strategy l: synthetic pathway of tetra-substitution of the NDA core and its derivatives through esterification, condensation and imidization, in sequence. 


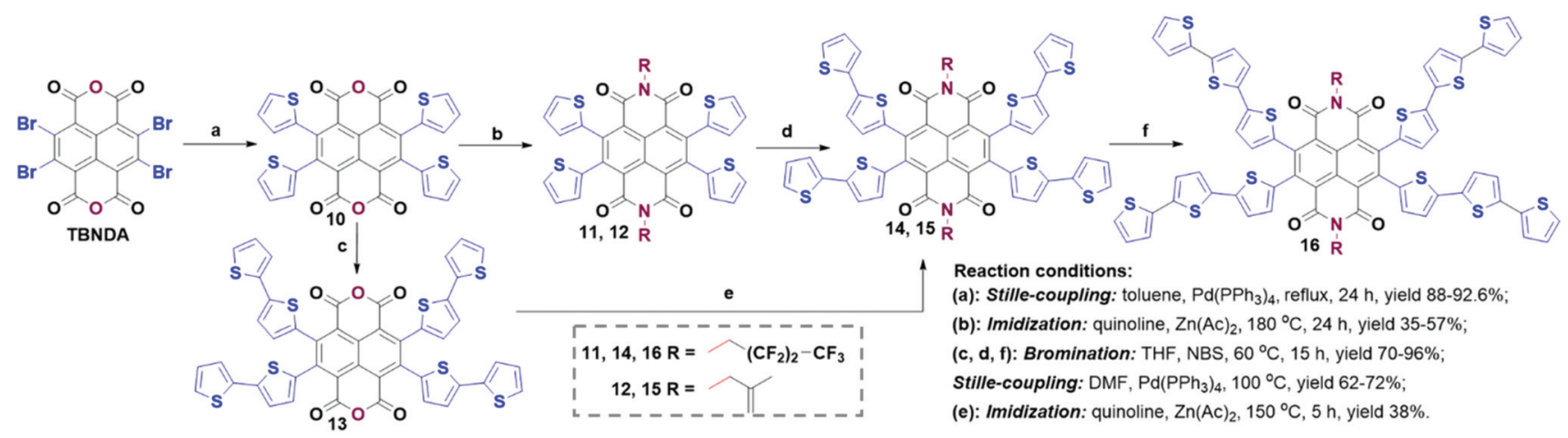

Scheme 3 Strategy II: the synthetic routes to star-shaped thiophene-NDI derivatives by direct core-functionalization of TBNDA.

$\mathrm{CH}_{2} \mathrm{Cl}_{2}$ solution. After sequential condensation and imidization, the targeted $\mathbf{8}$ and $\mathbf{9}$ were obtained as red powders. ${ }^{29} \mathrm{~A}$ similar annulation process provides more possibilities to expand the flanking TBNDI core, whereas it could not be replicated in tetra-ethynyl-substituted TBNDI.

\subsection{Strategy II: direct core-functionalization of TBNDA}

To the best of our knowledge, the examples of direct corefunctionalization of TBNDA are rare due to its poor solubility, tedious separation, and low yield. However, there are still some successful research studies. For example, as shown in Scheme 3, a soluble red compound $\mathbf{1 0}$ was obtained after the functionalization of TBNDA with four thiophene groups. Four substitutions were performed at 2, 3, 6 and 7 positions of TBNDA by Stille-coupling with 2-tributylstannyl-thiophen. With $\mathbf{1 0}$ as an intermediate, two synthetic routes could be conducted: one is to perform imidization first to give $\mathbf{1 1}$ and $\mathbf{1 2}$, followed by Stille-coupling reactions with 2-tributylstannylthiophen to form 14, 15 or 16 . The other one is to conduct Stille-coupling reactions with 2-tributylstannyl-thiophene first to produce 13, followed by imidization to give 14 and 15 . Additionally, $\mathbf{1 2}$ and $\mathbf{1 5}$ could act as copolymerizing agents to bind onto the other polymer chains due to the presence of 2-methylallyl groups. ${ }^{20,30}$

\subsection{Strategy III: synthesis of TBNDI precursor}

Introducing substituents onto the $\mathrm{N}$ atoms of imide is a common way to realize the chemical modification of TBNDA. The longitudinal $N$-substitution chains decide the solubility, molecular aggregation behavior, and the ability to form highquality films for broad applications in organic semiconducting devices. Besides, the electrophilic imide carbonyl groups have a significant effect on the polarization of $\pi$-systems, leading to a low $\pi$-electron density and high $\pi$-acidity. Zhu et al. found that some amino-substituted byproducts were very difficult to be purified or isolated throughout the whole imidization reaction between TBNDA and $n$-octylamine in acetic acid, due to the large conjugated structure and strong electrophilic ability of four Br atoms. Fortunately, an efficient methodology was finally explored at a yield of $31.4 \%$ with TBNDA as the starting material, where TBNDA refluxed with 4 -eq. of $n$-octylamines in
HOAc for $30 \mathrm{~min}$, followed by isolating the intermediate product and reacting with excess $\mathrm{PBr}_{3}$ in refluxing toluene. ${ }^{14}$ Later on, a modified synthetic method was presented, where a single solvent and mild temperature were used, and a whopping $72 \%$ yield was achieved. ${ }^{31}$ However, Würthner et al. could prepare the desired TBNDI by reacting TBNDA with nucleophilic 2,6-diisopropylaniline in glacial HOAc for $6 \mathrm{~h}$ at $120{ }^{\circ} \mathrm{C} .{ }^{15}$ The less nucleophilic arylamine reagent was essential to decrease the nucleophilic substitution of bromine atoms. This synthetic condition was further confirmed and optimized by the Facchetti group in 2013, and a high yield (96\%) of TBNDI was achieved by using 3-eq. of 2-octyldodecylamine in mixed solvents of $o$-xylene and propionic acid at $140{ }^{\circ} \mathrm{C}$ for $2 \mathrm{~h}^{21}$ However, Govindaraju et al. followed the procedure with 3 eq. of $n$-octylamine in glacial HOAc at $130{ }^{\circ} \mathrm{C}$ for $6 \mathrm{~h}$ to give the desired products only in $>10 \%$ yield, together with major ringopening products and a trace of core-substituted products. ${ }^{22}$ Finally, the targeted product was synthesized in good yield by two-step synthesis reported by Zhu et al. ${ }^{14}$ Besides, a microwave-assisted reaction was performed at $100 \mathrm{~W}$ for $45 \mathrm{~s}$ and then at $35-50 \mathrm{~W}$ for $30 \mathrm{~min}$ to maintain $150{ }^{\circ} \mathrm{C}$ in an open reaction vessel, to give a better yield $(65 \%)$ with a reduced amount of byproducts due to the complete or partial reductive dehalogenation..$^{32}$ All the reaction conditions are summarized in Table 2.

Table 2 The summary of various synthetic conditions for producing TBNDI via imidization

\begin{tabular}{|c|c|c|}
\hline Reaction conditions & Yield & Ref. \\
\hline $\begin{array}{l}\text { (1) } n \text {-Octylamine, HOAc, reflux, } 30 \mathrm{~min} \\
\text { (2) Excess } \mathrm{PBr}_{3} \text {, toluene, reflux, } 12 \mathrm{~h}\end{array}$ & $31.4 \%$ & 14 \\
\hline $\begin{array}{l}\text { (1) } n \text {-Octylamine, } \mathrm{CH}_{2} \mathrm{Cl}_{2} \text { or THF, reflux } \\
\text { (2) } \mathrm{PBr}_{3}, \mathrm{CH}_{2} \mathrm{Cl}_{2} \text {, reflux }\end{array}$ & $72 \%$ & 31 \\
\hline 2,6-Diisopropylaniline, HOAc, $120^{\circ} \mathrm{C}, 6 \mathrm{~h}$ & $29 \%$ & 15 \\
\hline $\begin{array}{l}\text { 2-Octyldodecylamine, } o \text {-xylene, propionic acid, } 140^{\circ} \mathrm{C} \text {, } \\
2 \mathrm{~h}\end{array}$ & $96 \%$ & 21 \\
\hline $\begin{array}{l}\text { (1) Microwave, } 100 \mathrm{~W}, 45 \mathrm{~s} \\
\text { (2) Microwave, } 35-50 \mathrm{~W}, 30 \mathrm{~min}, 150{ }^{\circ} \mathrm{C}\end{array}$ & $65 \%$ & 32 \\
\hline
\end{tabular}




\subsection{Core-annulated TBNDIs}

Core-functionalization through the replacement of halogen atoms with nucleophiles is the most efficient method to produce a broad range of TBNDI derivatives with various optical and electrochemical properties. Typically, Pd-catalyzed coupling reactions have been widely applied to link the central NDA core and versatile functionalized reagents together to construct large polycyclic aromatic analogues. ${ }^{33-38}$ These novel TBNDI derivatives could be divided into several categories, including heterocyclic annulations (S-, N-, S/N- and $\mathrm{O} / \mathrm{N}$-containing heterocycles), carbocyclic annulations or the fusion with $\mathrm{N}$-heterocycles, bis-TBNDI core analogues, and non-annulated TBNDI cores.

\subsubsection{S-Containing heterocycles}

Unilateral annulation. The incorporation of S-containing heterocycles becomes an efficient way to realize the annulation with the NDA core as well as to enhance intermolecular interactions. The HOMO and LUMO energy levels of the as-prepared compounds could be finely tuned to promote the electron injection and air-stable electron transport. Unilateral S-containing heterocyclic TBNDI derivatives through one-side nucleophilic aromatic substitution (SNAr) reactions are rare. Although different reaction conditions have been tried, Gao and co-workers still failed to produce unilateral products by using dicyano-substituted S-containing reagent I (Scheme 4) and TBNDI as starting materials. ${ }^{39 a}$ According to their trials and previous findings, they figured out that the unilateral S-containing heterocycle might be more electron-deficient than unreacted TBNDI, which made the second-step SNAr reaction more prone to directly forming the bilateral-annulation product instead of staying in the stage of the unilateral annulation intermediate. To avoid a bilateral annulation reaction, a less reactive nucleophilic reagent (cyano-containing reagent II, Scheme 4) was dropwise added into the THF solution of TBNDI at r.t., and finally, the unilateral annulation product was harvested in a moderate yield (36\%). ${ }^{40}$ The remaining bromine groups could be sequentially reduced in the presence of $\mathrm{NaBH}_{4}$ and Pd-catalyst or performed other modification reactions to construct new $\pi$-conjugation materials. Additionally, another method to produce unilateral sulfur-heterocycle fused annulation from mono- or di-bromo substituted NDI (MBNDI and DBNDI) has been reported by Gao et al. ${ }^{39 a}$ It's worth mentioning that the reaction processes of MBNDI or DBNDI with reagent I are different from that of TBNDI. Thus, the persuasive mechanism was proposed that an intermolecular nucleophilic attack and further a Michael addition reaction proceeded, followed by an oxidative aromatization process to afford the final products. For the monolateral derivatives from DBNDI, the reactive site of bromo provided more possibilities for ambipolar OSCs with fine-tuned energy levels. ${ }^{39 b}$

Symmetric bilateral annulation. The formation of bilateral S-containing heterocycle-fused TBNDIs has been widely reported via the SNAr reaction. As listed in Table 3, the representative centrosymmetric compounds 17-27 were obtained in good yields under mild reaction conditions. Typically, the corresponding structures of S-containing nucleophilic reagents are provided in Scheme 4, marked as I to XI. ${ }^{31,41-48}$ The reagents VII, VIII, IX and $\mathrm{X}$ are zinc salts prepared from different dithiols, while reagents VI and XI could react with $\mathrm{NaH}$ and $\mathrm{CS}_{2}$ to afford the corresponding sodium salts. Among these bilaterally annulated products, compound 17, as a promising n-channel organic semiconductor, has drawn much attention due to its potential value for organic electronic applications. Compound $\mathbf{1 7}$ was first synthesized in 50\% yield by stirring TBNDI and reagent I at $50{ }^{\circ} \mathrm{C}$ for $1 \mathrm{~h} .{ }^{41}$ Later on, the one-pot synthesis of compound $\mathbf{1 7}$ was developed directly from

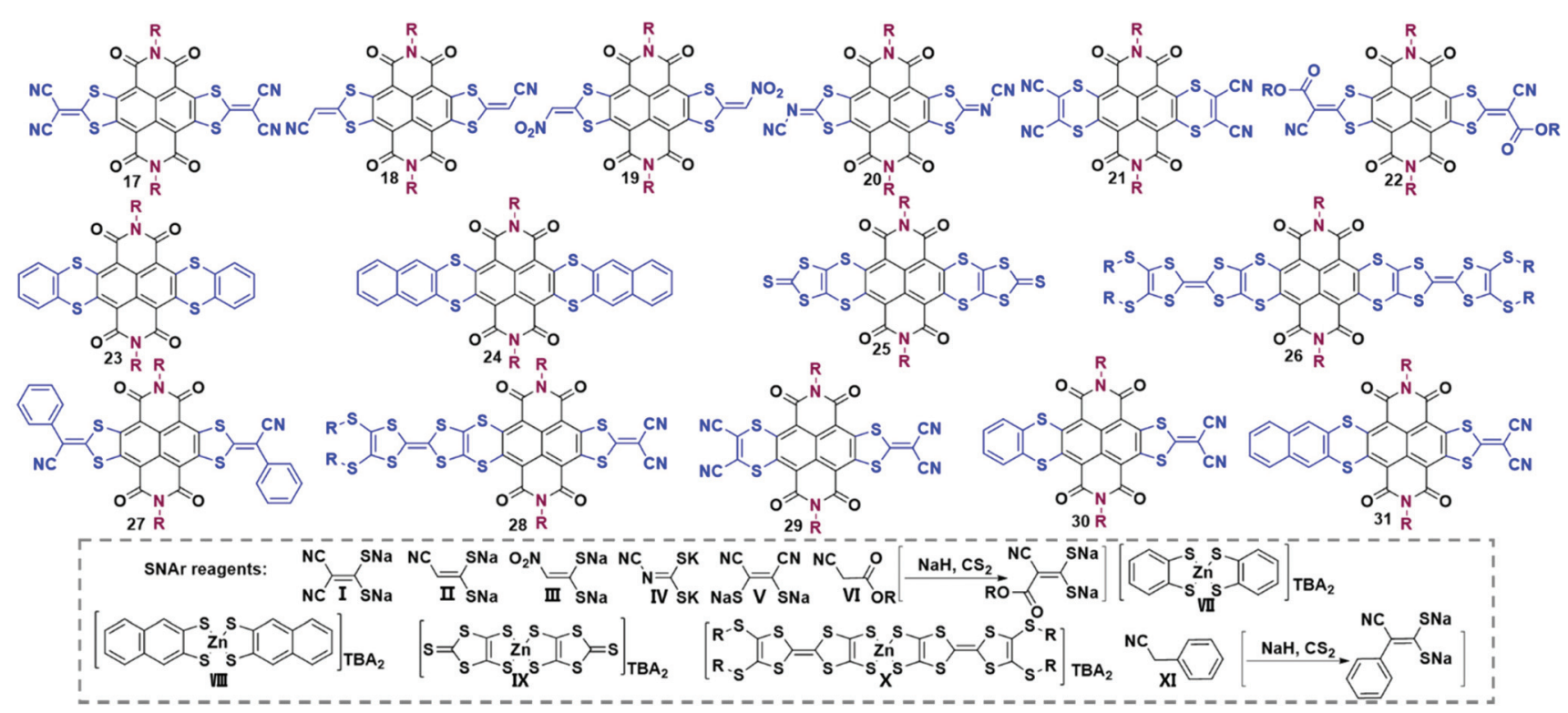

Scheme 4 Molecular structures of compounds $17-31$ and S-containing SNAr reagents (I to XI). 
Table 3 Synthetic conditions for symmetric and asymmetric bilateral $\mathrm{S}$-containing heterocycle fused NDIs

\begin{tabular}{|c|c|c|c|c|}
\hline Compound & $\begin{array}{l}\text { S-Containing } \\
\text { reagent }\end{array}$ & Reaction conditions & Yield & Ref. \\
\hline \multirow[t]{2}{*}{17} & I & $\mathrm{THF}, 50^{\circ} \mathrm{C}, 1 \mathrm{~h}$ & $50 \%$ & 41 \\
\hline & I & $\begin{array}{l}\text { DMF, r.t., } 50^{\circ} \mathrm{C}, 6 \mathrm{~h} \\
\text { (one-pot synthesis } \\
\text { from TBNDA) }\end{array}$ & $\begin{array}{l}28 \%- \\
55 \%\end{array}$ & 42 \\
\hline 18 & II & THF, r.t., 3 h & $90 \%$ & 43 \\
\hline 19 & III & THF, r.t., $1 \mathrm{~h}$ & $30 \%$ & 44 \\
\hline 20 & IV & $\mathrm{THF}, 50^{\circ} \mathrm{C}, 1 \mathrm{~h}$ & $64 \%$ & 31 \\
\hline 21 & $\mathrm{~V}$ & $\mathrm{THF}, 30^{\circ} \mathrm{C}, 30 \mathrm{~min}$ & $35 \%$ & 45 \\
\hline 22 & VI & $\begin{array}{l}\text { (1) } \mathrm{NaH}, \mathrm{CS}_{2}, \mathrm{THF} \text {, } \\
0-5{ }^{\circ} \mathrm{C} \\
\text { (2) R.t., } 5 \mathrm{~h}\end{array}$ & $73 \%$ & 44 \\
\hline 23 & VII & THF, r.t., 2 h & $98 \%$ & 46 \\
\hline 24 & VIII & THF. r.t., 2 h & $86 \%$ & 46 \\
\hline 25 & IX & THF, r.t., $1 \mathrm{~h}$ & $72 \%$ & 47 \\
\hline 26 & $\mathrm{X}$ & THF, r.t., $3 \mathrm{~h}$ & $35 \%$ & 48 \\
\hline 27 & $\mathrm{XI}$ & $\begin{array}{l}\text { (1) } \mathrm{NaH}, \mathrm{CS}_{2}, \mathrm{THF} \text {, } \\
5-10^{\circ} \mathrm{C}, 30 \mathrm{~min} \\
\text { (2) R.t., } 5 \mathrm{~h}\end{array}$ & $91 \%$ & 44 \\
\hline 28 & $X+I$ & $\begin{array}{l}\text { (1) X, THF, r.t., } 1 \mathrm{~h} \\
\text { (2) I, THF, r.t., } 3 \mathrm{~h}\end{array}$ & $26 \%$ & 48 \\
\hline 29 & $\mathrm{I}+\mathrm{V}$ & $\begin{array}{l}\text { (1) I, V, THF, }-10^{\circ} \mathrm{C} \\
\text { (2) R.t., } 2.5 \mathrm{~h}\end{array}$ & $70 \%$ & 44 \\
\hline 30 & $\mathrm{VII}+\mathrm{I}$ & $\begin{array}{l}\text { (1) VII, THF, r.t., } \\
30 \mathrm{~min} \\
\text { (2) I, THF, r.t., } 2 \text { h }\end{array}$ & $59 \%$ & 46 \\
\hline 31 & $\mathrm{VIII}+\mathrm{I}$ & $\begin{array}{l}\text { (1) VIII, THF, r.t., } \\
30 \mathrm{~min} \\
\text { (2) I, THF, r.t., } 2 \text { h }\end{array}$ & $38 \%$ & 46 \\
\hline
\end{tabular}

TBNDA through a feasible SNAr and then an imidization reaction under mild conditions. ${ }^{42}$ Gao et al. also found that compound 17 could be produced as the only product from DBNDI in DMF rather than dichloromethane $\left(\mathrm{CH}_{2} \mathrm{Cl}_{2}\right)$ at r.t., indicating that the solvent polarity acts as a significant factor for the reaction between hydrophilic salts and hydrophobic molecules. ${ }^{39 a}$ The lower yield of $27 \%$ was attributed to the impaired reactivity of the dibromo group of DBNDI.

Asymmetric bilateral annulation. Few cases of asymmetric bilateral annulation have been presented in the literature. ${ }^{44,46,48}$ As listed in Table 3, compounds 28-31 were synthesized sequentially or simultaneously using the two S-containing reagents at low temperature. Compounds 28, 30 and 31 were obtained in decent yields by the first-step reaction between equivalent TBNDI and the respective zinc salts, followed by the addition of reagent $\mathrm{I}$ and sequential stirring at $\mathrm{r}$. t. to yield targeted products. ${ }^{46,48}$ The preparation of 29 could be realized by adding reagents $\mathrm{I}$ and $\mathrm{V}$ in one portion to the TBNDI solution at $-10{ }^{\circ} \mathrm{C}$, followed by additionally stirring at r.t. for $2.5 \mathrm{~h} .{ }^{44}$ Given that the asymmetric bilateral annulation with a S-containing heterocycle could be successfully synthesized with zinc salts or other reagents, there remains plenty of room for chemical modification of TBNDI to develop new good performance OSCs.

Further functionalization. Some of the bilateral S-containing heterocycles could be further functionalized (Scheme 5). For example, due to the existence of two reactive sites in the symmetric bilateral annulation compound 18, it could be further halogenated to afford 32. The introduction of $\mathrm{Br}$ atoms on the extended NDI skeleton could enable the construction of functional analogues through a Pd-catalyzed cross-coupling reaction, where small molecules $(\mathbf{3 3}, \mathbf{3 4}, \mathbf{3 5})$ and donor-acceptor (D/A) copolymers (36 and 37 ) were synthesized to show nearinfrared (NIR) optical absorptions. ${ }^{43,49}$ An example of six-membered compound 21 was converted into a mixture of five-membered 38 (syn) and 39 (anti) isomers. ${ }^{45}$ The S-rich compound 25 could be oxidized into the oxygen-containing compound $\mathbf{4 0}$ with $\mathrm{Hg}(\mathrm{OAc})_{2}$ at room temperature. ${ }^{47}$ Thereby, the functionalization of S-containing heterocycles could be considered as a viable protocol to prepare more S-containing electron-transporting materials.

\subsubsection{N-Containing heterocycles}

Unilateral annulation. As shown in Scheme 6, unilateral annulation derivatives (41-46) could be readily prepared by refluxing TBNDI with ortho diamines in DMF at $120-135{ }^{\circ} \mathrm{C}$ for a while (15-60 min). The high yield (70-90\%) of 41-46 might be attributed to the weak nucleophilicity of anilines and the deactivating effect of two amino groups to preclude further tetra-substitution. ${ }^{50-52}$ Chi et al. found that the debrominated side products were always accompanied by compound $\mathbf{4 7}$ when the reaction was conducted in DMF at $135{ }^{\circ} \mathrm{C}$, leading to an unseparated product with a poor yield. To address this issue, the same group employed $\mathrm{CHCl}_{3}$ to replace DMF and conducted the reaction at r.t. to push the yield to $83 \%{ }^{53}$ With the usage of less nucleophilic diamine reagents, SNAr reactions could only take place in alkaline conditions (excess KOAc or

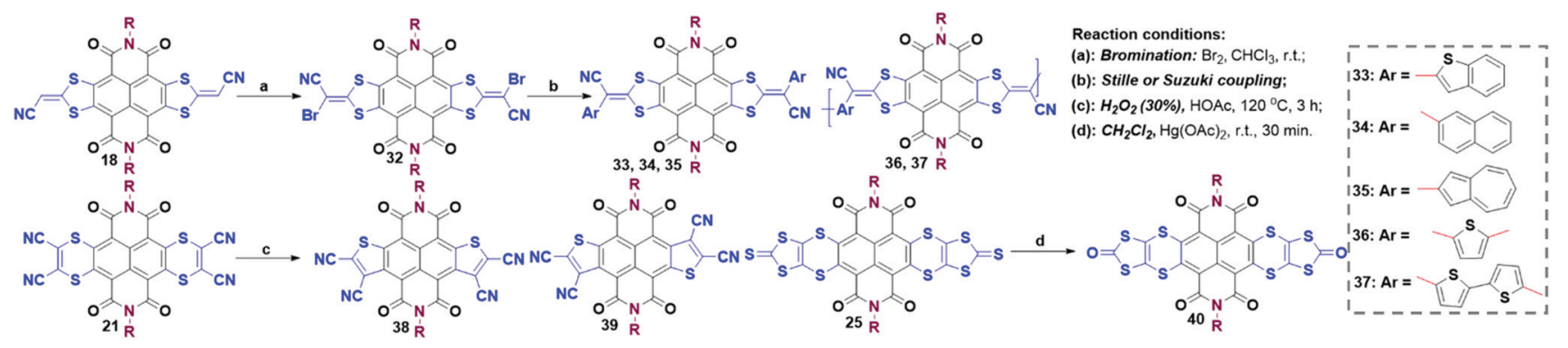

Scheme 5 Synthetic strategies for further functionalization of bilateral S-containing heterocycles. 


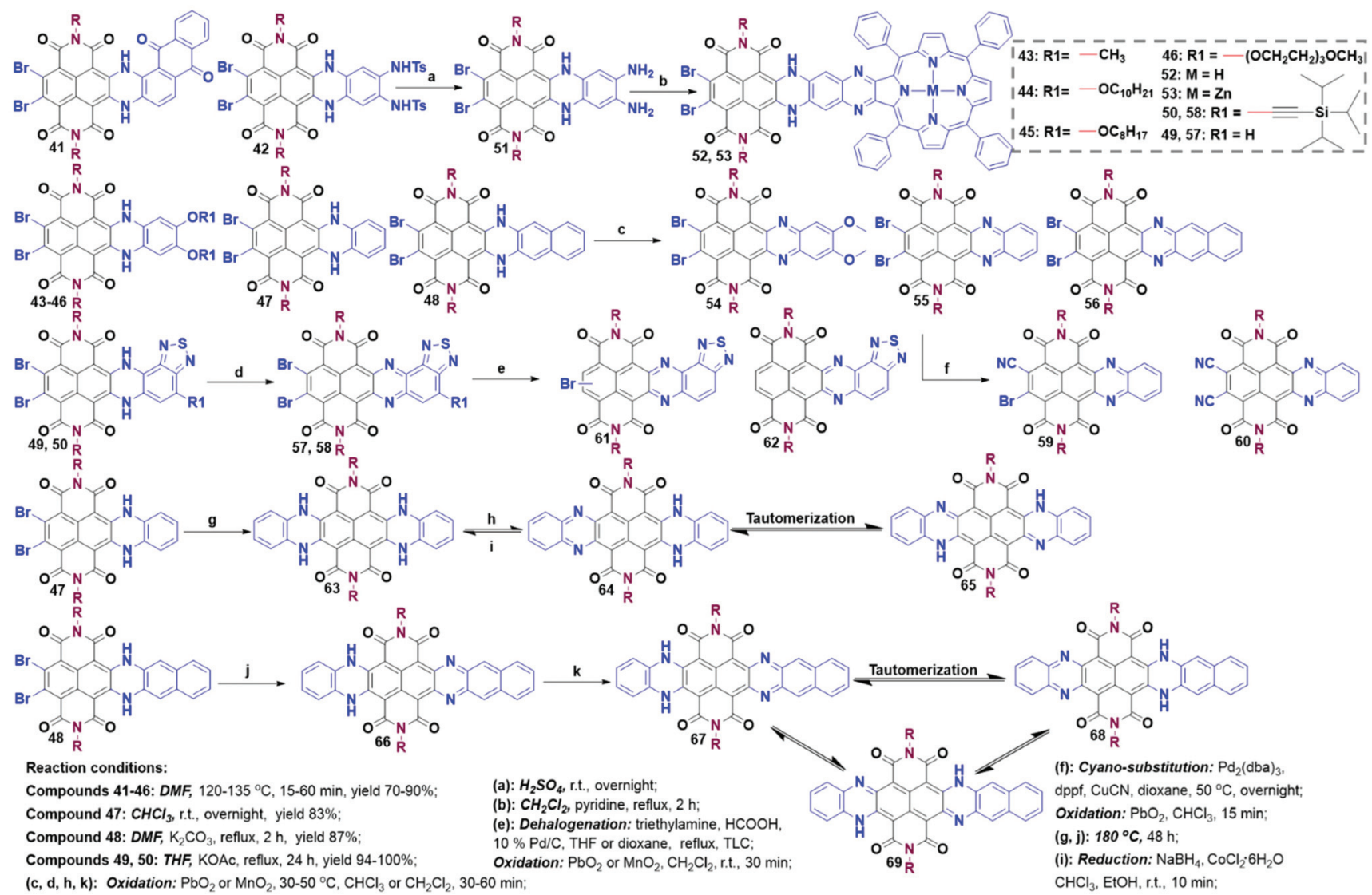

Scheme 6 Structures and synthesis of unilateral and bilateral N-heterocycle derivatives.

$\mathrm{K}_{2} \mathrm{CO}_{3}$ ) and afford 48, 49 and 50 (Scheme 6). ${ }^{54,55}$ Although the remaining two aryl bromide groups could not react with extra diamines under the same conditions, it could still provide more possibilities to modify the NDA core with different functional groups at $\mathrm{Br}$ sites.

Symmetric and asymmetric bilateral annulation. During the preparation of 49 , trace bilateral annulation compounds as two isomers were observed and separated. ${ }^{55}$ Under harsh conditions $\left(180{ }^{\circ} \mathrm{C}\right.$ and more than two days), those unilaterally annulated compounds could further react with extra diamines to give 63 and 66 in low yields of $28 \%-31 \% .{ }^{54}$ Considering the tedious synthesis, the research on bilateral N-containing annulation as well as further modification received less attention.

Further modification. These N-containing heterocyclic TBNDIs could be explored into highly sophisticated structures. For example, compounds 52 and 53 were prepared via constructing a rigid aromatic diaza bridge to link a free- or zinctetraphenylporphyrin. The two donor-bridge-acceptor entities exhibited a large electron coupling interaction and weak negative driving force. ${ }^{56}$ Most of the N-heterocyclic TBNDIs containing $\mathrm{NH}$ groups showed weak aromaticity across the entire core, which might reduce the efficiency of charge-transfer. Therefore, further deprotonation of $\mathrm{NH}$ groups in mono- and di-annulation was carried out. Quantitative unilateral oxidation products $\mathbf{5 4}, \mathbf{5 5}$ and $\mathbf{5 7 ,} 58$ were easily obtained in the presence of $\mathrm{PbO}_{2}$ or $\mathrm{MnO}_{2}$ under mild conditions, whereas the oxidation reaction of $\mathbf{4 8}$ required a higher temperature. The asobtained oxidation product 56 could be converted into 48 in silica gel during the purification process, due to its intrinsic low LUMO level. A cyanation reaction of $\mathbf{5 5}$ was realized in the presence of $\mathrm{Pd}_{2}(\mathrm{dba})_{3}$, dppf and $\mathrm{CuCN}$ in dioxane, where mono- and di-cyanated intermediates with the reduction of the imine bonds (59 and 60) could be harvested in the yields of $30 \%$ and $24 \%$, respectively. Note that $\mathbf{6 0}$ was very sensitive to silica gel or water due to its ultrahigh electron affinity. ${ }^{53}$ These results indicate that the stability of the mono-annulated oxidation products is closely correlated with their LUMO energy levels. A dehalogenation reaction of $\mathbf{5 7}$ could happen in the presence of $\mathrm{Pd} / \mathrm{C}$ catalyst, formic acid, and triethylamine. Furthermore, $\mathbf{6 1}$ and $\mathbf{6 2}$ could be prepared through the removal of one or two bromine atoms in $\mathbf{5 7}$ together with the reduction of the imine bonds, then followed by the oxidation. For bi-annulation compound $\mathbf{6 3}$, the oxidation reaction was accomplished smoothly under mild conditions to afford a mixture of two tautomers (benzenoid-type compound 64 and quinonoid-type compound 65). The tautomers could be swiftly reverted to 63 using reductive agents $\left(\mathrm{NaBH}_{4}\right.$ and $\left.\mathrm{CoCl}_{2}\right)$. The oxidation product of $\mathbf{6 6}$ generated three tautomers, including two benzenoid-type products 67 and 68, and a quinonoid-type product 69. 

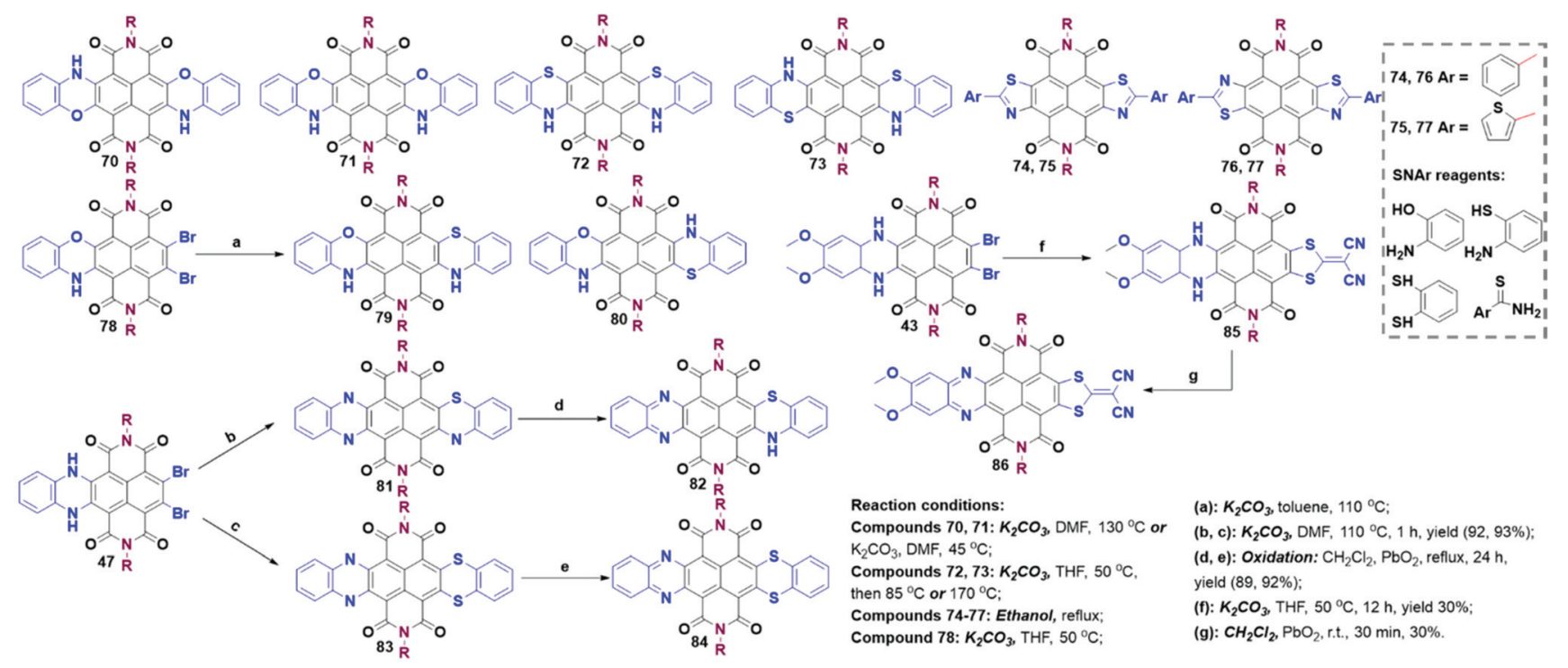

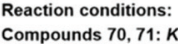
Compo $70,71: \mathrm{K}_{2} \mathrm{CO}_{3}$ DMF $130^{\circ} \mathrm{C}$ or $\mathrm{K}_{2} \mathrm{CO}_{3}, \mathrm{DMF}, 45^{\circ} \mathrm{C}$ Compounds 72, 73: $\mathrm{K}_{2} \mathrm{CO}_{3}, \mathrm{THF}, 50^{\circ} \mathrm{C}$ then $85^{\circ} \mathrm{C}$ or $170^{\circ} \mathrm{C}$; Compounds 74-77: Ethanol, reflux Compound 78: $\mathrm{K}_{2} \mathrm{CO}_{3}, \mathrm{THF}, 50^{\circ} \mathrm{C}$;

(f): $\mathrm{K}_{2} \mathrm{CO}_{3}, \mathrm{THF}, 50^{\circ} \mathrm{C}, 12 \mathrm{~h}$, yield $30 \%$
(g): $\mathrm{CH}_{2} \mathrm{Cl}_{2}, \mathrm{PbO}$, r.t. $30 \mathrm{~min}, 30 \%$.

Scheme 7 Structures and synthesis of $\mathrm{S} / \mathrm{N}$ - and $\mathrm{O} / \mathrm{N}$-containing heterocycle derivatives.

\subsection{3 $\mathrm{S} / \mathrm{N}-$ and $\mathrm{O} / \mathrm{N}$-containing heterocycles}

Symmetric bilateral heterocyclic annulation. As displayed in Scheme 7 , several $\mathrm{S} / \mathrm{N}$ and $\mathrm{O} / \mathrm{N}$-containing bilateral annulations with the coexistence of anti- and syn-isomers (70-77) were synthesized. Solvents and reaction temperature strongly influenced the selectivity and yields of these anti- and synisomers, particularly, the synergetic effects of polarity variance in solvents, hydrogen bonding, and the changed nucleophilicity of $\mathrm{N}$ and $\mathrm{S}$. Note that only anti-isomers could be oxidized into the fixed quinonoid-type structures. Besides, in contrast to 63 , the incorporation of $\mathrm{O}$ or $\mathrm{S}$ could effectively change the tautomerization during the oxidation of $\mathrm{NH}$ moieties. Thus, quinonoid-type structures with impressive stability and notable electron-transporting capabilities were readily obtained. ${ }^{57}$ This strategy was also deemed as an efficient way to isolate and purify the syn- and anti-isomers. Compounds 74-77 fused with dithiazole containing a coplanar skeleton and an enhanced conjugation length could also be synthesized with DBNDI as a starting compound without any solvents and metallic catalysts. ${ }^{58}$

Asymmetric bilateral heterocyclic annulation. A series of fiveand six-membered asymmetric annulated products were attained by the condensation reaction between TBNDI and $o$-phenylenediamine, thiophene-2-carbothioamide, 1,2-benzenedithiol or 2-aminothiophenol. ${ }^{59-61}$ Firstly, the mono-annulation process was involved to afford the intermediates 78, 47 and 43 (Scheme 7). Further asymmetric bilateral annulations and subsequent oxidations could produce 79-86. All the oxidation process took place in a finely mild way to give a quantitative yield. Intriguingly, the oxidation reaction of compound 81 could only smoothly happen on the two NH moieties at the same side, which produced benzenoid-type product $\mathbf{8 2}$ rather than a quinonoid-type product. ${ }^{61}$ The deactivation of the 6,7positions of $\mathbf{4 3}$ was ascribed to the introduction of a strong electron-donor group. Thus, the asymmetric core-extended compound 85 with a D-A configuration was synthesized in a moderate yield (30\%) through the reaction between $\mathbf{4 3}$ and the corresponding S-containing nucleophilic reagent. A high-yield debromination reaction was observed during an attempt to oxidize 43, which might be caused by the twisted aromatic plane relating to the neighboring bromine groups. ${ }^{60}$

\subsubsection{Carbocyclic annulation and heterocycle-fused carbo-} cyclic annulation

Symmetric bilateral carbocyclic annulation. The bilateral carbocycle-extensions were realized through the formation of $\mathrm{C}-\mathrm{C}$ bonds in cross-coupling reactions in the presence of organometallic reagents such as zirconacyclopentadiene reagents ( $\mathbf{Z r}$ 1-Zr-5), which were prepared from the reaction between alkynes and zirconocene in anhydrous toluene. The coupling reaction between metallacyclopentadiene and the corresponding organometallic reagents in the presence of copper chloride $(\mathrm{CuCl})$ produced $\mathbf{8 7 - 9 0}$ (Scheme 8) with a moderate yield. ${ }^{62}$ However, the attempts of further dehydrogenation of cyclohexene-fused compound $\mathbf{8 9}$ to generate large acene imides were failed. ${ }^{63}$ Pd-Catalyzed double cross-coupling was also recognized as a straightforward method to develop TBNDI derivatives. The cross-coupling reactions between stannane reagents (Sn-1 and Sn-2) and TBNDI were conducted successfully in the presence of $\mathrm{Pd}\left(\mathrm{P}(t-\mathrm{Bu})_{3}\right)_{2}$ and $\mathrm{CsF}$ at $70{ }^{\circ} \mathrm{C}$ to afford new aromatic annulations 91 and $\mathbf{9 2}$.

Asymmetric heterocycle-fused carbocyclic annulation. Inspired by bilateral core-extensions, unilateral carbocyclic annulation was also developed with the N-heterocyclic compound $\mathbf{4 7}$ as a starting material. Specifically, the reaction between $\mathbf{4 7}$ and the respective zirconacyclopentadiene reagents ( $\mathbf{Z r - 3}$ and $\mathbf{Z r}-5)$ in refluxing toluene in the presence of 2-eq. of $\mathrm{CuCl}$ gave 93 (yield: 24\%) and 95 (yield: 45\%). Subsequent dehydrogenative aromatizations were conducted in 5 min to give 94 and 96 


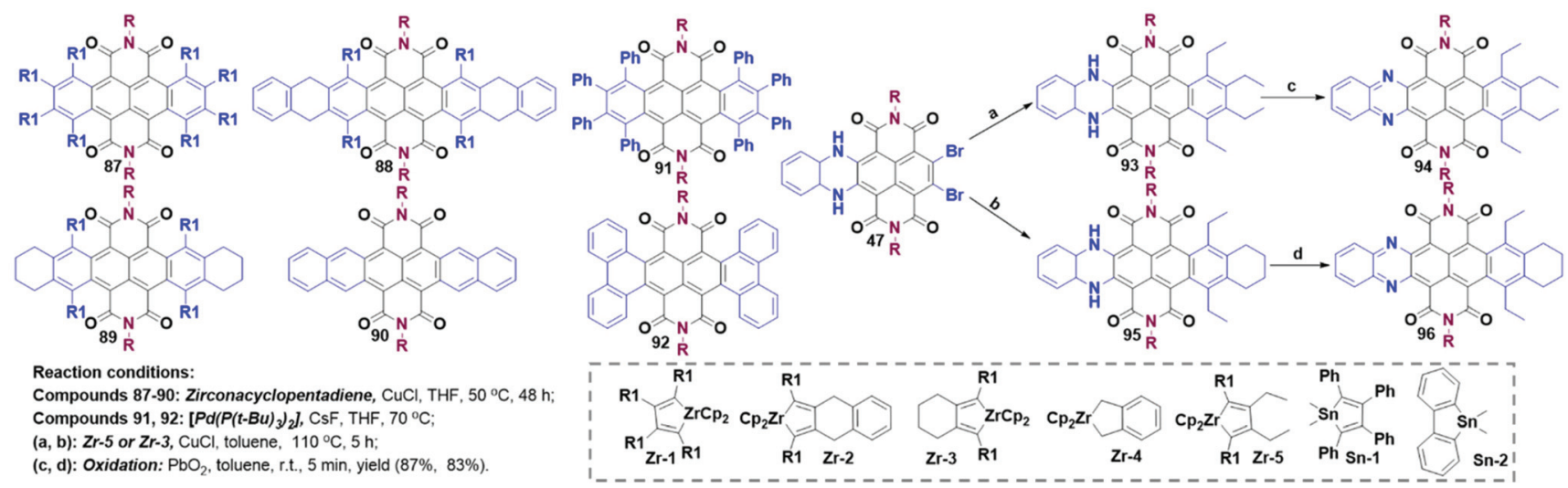

Scheme 8 Synthetic routes of carbocyclic annulation and heterocycle-fused carbocyclic annulation.

with high yields ( $87 \%$ and $83 \%)$. Such a strategy to fuse the $\mathrm{N}$-heteroarenes and carbocyclic rings together opens a broad avenue to construct new TBNDI-based electron-transporting systems.

\subsection{Bis-TBNDI core analogues}

Bis-TBNDI analogues composed of two NDI cores are seldom reported, due to the rigorous requirement of elaborated molecular design and synthetic routes. Only a limited number of representative examples with core-expanded heterocyclic rings and red-to-NIR absorptions are summarized here. According to different synthetic routes, these examples are separated into two parts: one type is to carry out unilateral annulation first before the formation of bis-TBNDI systems, and the other is to construct bis-TBNDI systems first, followed by lateral annulations.

2.6.1 From lateral annulation to bis-TBNDI. In Scheme 9, asymmetric bilateral S-containing annulation compound $\mathbf{9 7}$ was readily prepared by the one-step SNAr reaction in $34 \%$ yield. A brominated intermediate was conveniently formed in a yield of $88 \%$ via treating 97 with $\mathrm{Br}_{2}$. However, the bis-TBNDI compound was failed to be obtained by the straightforward oxidative coupling reactions with commonly-used synthetic conditions, for instance directly reacting with distannanemediated reagents. Fortunately, bis-TBNDI compound 98 was finally achieved in a good yield with the aid of catalysts $\mathrm{Pd}$ $(\mathrm{OAc})_{2}$ and ${ }^{\mathrm{i}} \mathrm{Pr}_{2} \mathrm{EtN}$ in DMF. Besides, the model molecule 98 showed nonplanar molecular backbones with the intramolecular dihedral angle of $123^{\circ}$, calculated on the Gaussian 09 program at the B3LYP/6-31G(d,p) level. Such twisted molecular backbone structures endowed the bis-TBNDI derivatives with good solubility in organic solvents. ${ }^{40 a}$ Another work is to incorporate different end-capped groups at compound 18 precursor. By the reaction of one-side bromination and then Suzuki-Miyaura cross-coupling, intermediates 99, 100, and 101 were achieved in good yield. The three compounds were further modified to afford the corresponding bis-TBNDI derivatives 102, 103, and 104 through vinyl $\mathrm{C}-\mathrm{H} / \mathrm{C}-\mathrm{H}$ homocouplings in high yields of $85-94 \%$. All the dimeric products exhibited broad optical absorptions with narrow bandgaps and low-lying LUMO energies, revealing potential applications for ambient stable organic electronic devices. ${ }^{40 b}$

2.6.2 From bis-TBNDI to lateral annulation. Seven-ringexpanded bis-TBNDI intermediates and 9- or 11-membered $\mathrm{D} / \mathrm{A}$ derivatives have been reported in recent years. Zhao et al. found that a $1: 1$ condensation happened between TBNDI and benzene-1,2,4,5-tetraamine at $35{ }^{\circ} \mathrm{C}$. Only when the reaction temperature was raised to $80{ }^{\circ} \mathrm{C}$, seven-ring-expanded bisTBNDI intermediate 105 could be prepared as a deep green solid. Intriguingly, compound 105 containing a tetramine skeleton had a planar structure with NIR J-aggregation behaviors in aliphatic solvents. ${ }^{64}$ Only benzenoid-type product 106 was identified using NMR spectra after its oxidation. Moreover, 105 and 106 were hardly extended to larger $\pi$-conjugation backbones due to the deactivation of $\mathrm{C}-\mathrm{Br}$ bonds with the introduction of electron-donating $\mathrm{NH}$ groups. To avoid this issue, a new S-containing bis-TBNDI intermediate $\mathbf{1 0 7}$ was synthesized, which could be smoothly converted into eleven-membered compound 108 and nine-membered 109 by reacting with the S-containing nucleophile. ${ }^{65}$ In contrast to intermediates 105 and 107, the intermediate $\mathbf{1 1 0}$ was constructed via a one-pot tandem reaction, involving a primary SNAr attack and a sequent metal-free $\mathrm{C}-\mathrm{C}$ coupling reaction. Presumably, the first introduced electron-donating $\mathrm{NH}$ moiety activated its para- $\mathrm{CH}$ for the next intramolecular $\mathrm{C}-\mathrm{C}$ coupling reaction. This novel mechanism could explain the tandem process well, which might be utilized to guide the synthesis of more extended annulation based on the TBNDI core. Further functionalization (oxidation and SNAr) of intermediate 110 proceeded to produce $\mathbf{1 1 1}$ and $\mathbf{1 1 2}$ under the respective conditions, shown in Scheme 9. The quinoid-type compound $\mathbf{1 1 1}$ was swiftly reduced to intermediate $\mathbf{1 1 0}$ in a few seconds in the presence of $p$-phenylenediamine in $\mathrm{CHCl}_{3}$ at r.t. ${ }^{66}$

\subsection{Non-annulated TBNDI analogues}

A majority of non-annulated TBNDI derivatives consisting of mono-, di-, tri- and tetra-substitution have been invented as distinctive TBNDA synthons. Similar to the annulation reac- 


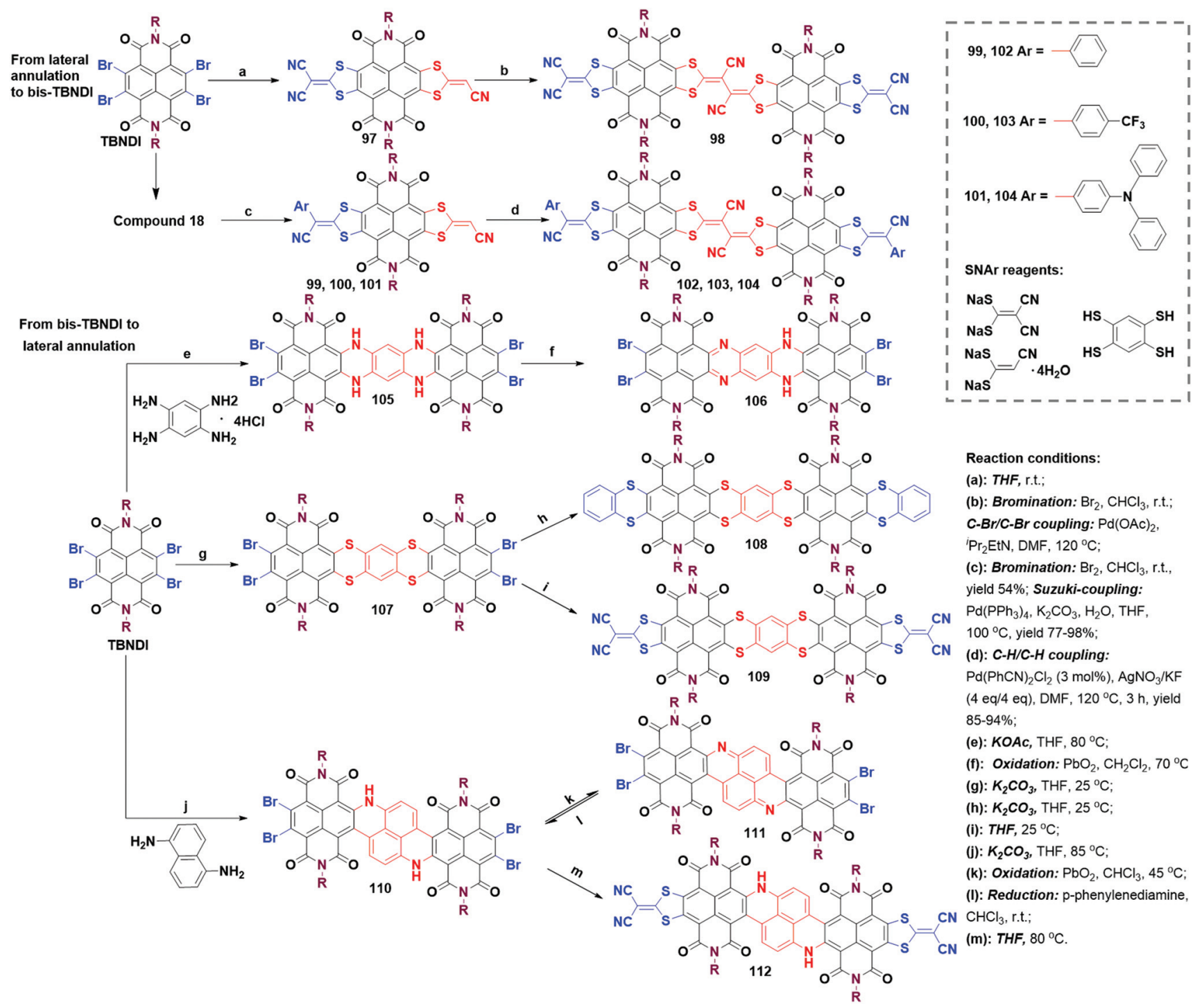

Scheme 9 Synthetic routes of symmetric bis-TBNDI analogues and their core-annulations.

tions, non-annulated analogues could also be obtained by the nucleophilic substitutions (i.e., N-, S-, or O-containing alkylsubstitution) or Pd-catalyzed cross-coupling reactions (tetraaryl- and tetraethynyl-substitution). The site selectivity of the above N-containing nucleophilic substitution reactions was provided more advantages to build more TBNDI blocks. Besides, some other functional strategies, such as dehalogenation, halogen-exchange and cyano-substitution, are also concisely summarized in this part.

2.7.1 Alkyl substitution. The alkyl-substituted TBNDI derivatives have been efficiently synthesized by the nucleophilic substitution reaction via utilizing alkoxy-, alkylthio-, or alkylamino-nucleophiles. In Scheme 10, the ring-closed compound 113 was produced by the reaction between TBNDI and ethylene diamine at $135{ }^{\circ} \mathrm{C}$ or a microwave-assisted protocol. ${ }^{32}$ Core-tetraamino-functionalized compound $\mathbf{1 1 4}$ could be obtained by refluxing TBNDI and alkylamino nucleophiles together in

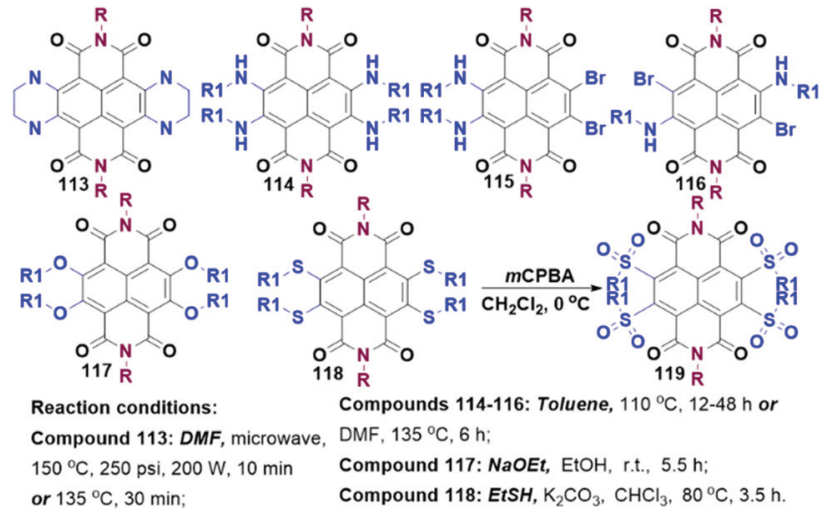

Scheme 10 Synthetic routes to alkyl substitution of TBNDI. 


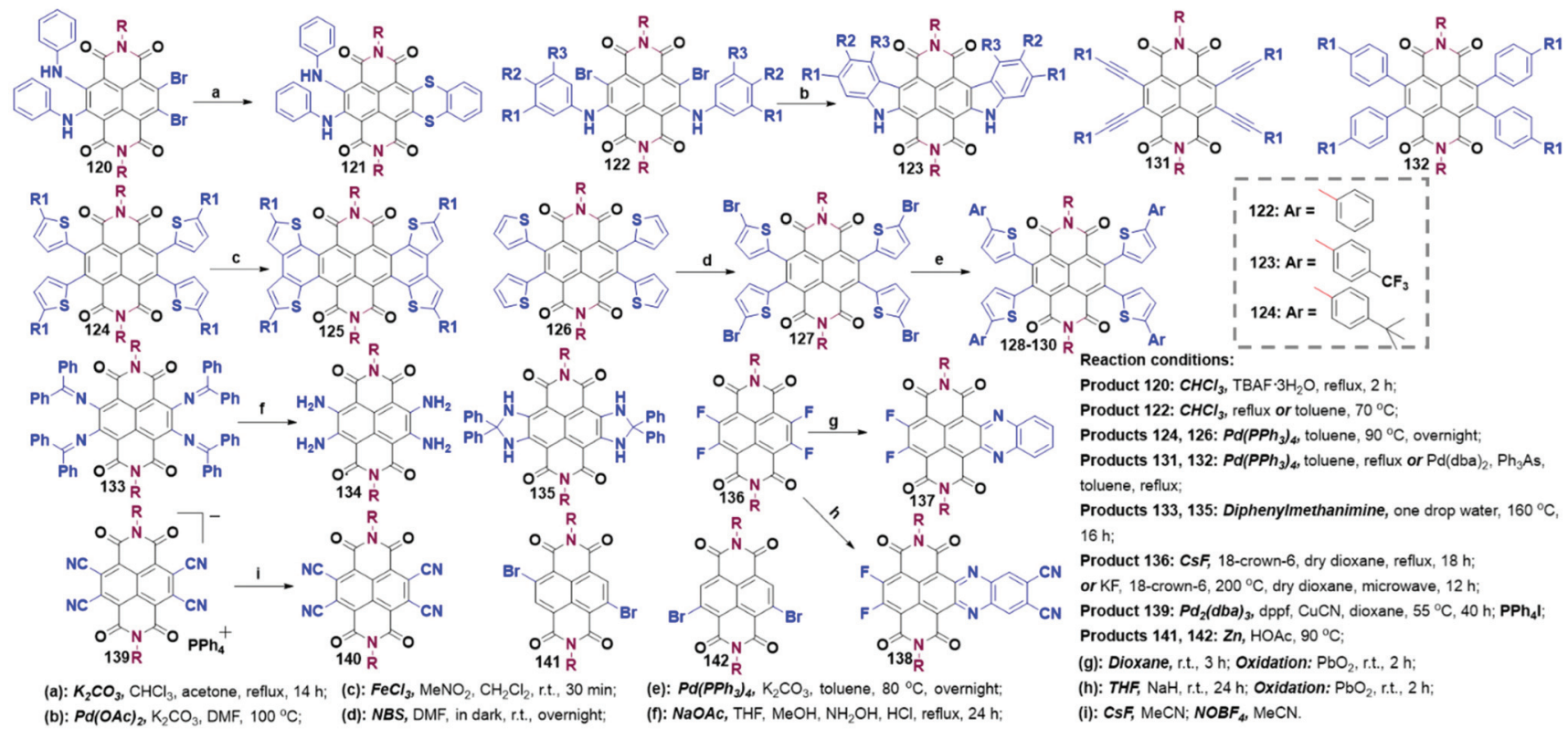

Scheme 11 Synthetic routes to other non-annulated TBNDI derivatives.

toluene or DMF, or through the reaction between TBNDA and alkylamino in a one-step process. ${ }^{22,23}$ Bilateral and unilateral diamino-substitution products $\mathbf{1 1 5}$ and $\mathbf{1 1 6}$ were prepared under modified reaction conditions with different equivalents of nucleophilic reagents. ${ }^{67}$ Tetraethoxy-substituted compound 117 was available under the alkaline reaction conditions involving sodium ethanolate at r.t., while tetraethylthio-substituted compound 118 was harvested in the presence of potassium carbonate in chloroform at $80{ }^{\circ} \mathrm{C} .{ }^{68}$ Furthermore, through the reaction between $\pi$-donating sulfide 118 and excess $m$-chloroperoxybenzoic acid $(m \mathrm{CPBA})$, the most $\pi$-acidic NDI derivative 119 with $\pi$-accepting sulfone groups and superior anion transport activity could be formed. ${ }^{69}$

2.7.2 Aryl and heteroaryl substitution. Aryl and heteroarylsubstituted TBNDI derivatives can be obtained through the nucleophilic reactions or Pd-catalyzed Stille cross-coupling reactions. The regioselectivity of nucleophilic substitutions and the further formation of expanded annulations have attracted a lot of interest. Würthner et al. found that the diamino-substituted regioselectivity of TBNDI was greatly influenced by the reaction solvents and additives in the presence of monodentate nucleophiles. For example, the existence or not of fluoride anion additives in the SNAr reaction under the same conditions gave different proportions of regioisomers. The reaction mechanism was speculated that the deprotonated intermediate was formed by the reaction between the monoamino-TBNDI intermediate and fluoride anions, leading to the sequential regioselective attack to give the preferential diamino-substituted product. On this basis, unilateral dianiline-substituted compound $\mathbf{1 2 0}$ was achieved with a high yield in the presence of tetrabutylammonium fluoride (TBAF) refluxing in $\mathrm{CHCl}_{3}$ for $2 \mathrm{~h}$. Compound 120, similar to the annulated compound $\mathbf{4 3}$, held the potential to expand the $\pi$-conjugated skeleton via further modification, for instance the formation of asymmetrical core-substituted compound $\mathbf{1 2 1}$ as shown in Scheme $11 .^{70}$ Bilaterally dianiline-substituted compound $\mathbf{1 2 2}$ was readily acquired through the regioselective nucleophilic substitution. The subsequent intramolecular arylation by $\mathrm{C}-\mathrm{C}$ cross-coupling afforded the syn-isomeric five-membered heterocyclic compound $\mathbf{1 2 3} .^{71}$

The non-annulated tetraaryl-, tetraethynyl- or tetrathiophene-substituted Stille coupling reaction could proceed with the Pd-catalyst, where the four substituents were restricted due to the inevitable steric constraints. In contrast to the tetrathiophthene-substituted TBNDI derivatives synthesized from TBNDA in Scheme 3, compounds $\mathbf{1 2 4}$ and $\mathbf{1 2 6}$ were originated from a 4-fold Stille coupling of TBNDI synthon with the respective yield of $63 \%$ and $56 \%$, respectively. Light-blocking bromination of compound $\mathbf{1 2 6}$ with NBS in DMF produced compound 127 in $87 \%$ yield, followed by a 4 -fold Suzuki coupling reaction to give 128-130 in a quantitative yield. Ferric chloride mediated oxidative cyclization of $\mathbf{1 2 4}$ was smoothly performed to give $\mathbf{1 2 5}$ in $80 \%$ yield, while $\mathbf{1 2 8 - 1 3 0}$ failed to complete the cyclization reaction even with the prolonged reaction time, different loading amounts of oxidants, and various reaction temperatures. ${ }^{72}$ The failures were speculated that the as-formed radical cations might delocalize when the electronrich thienyl groups were introduced as the substituents, which may lead to the deactivation of the radical cation, intermolecular coupling and chlorination. This result demonstrates that the oxidation potential of the reactants has a significant impact on the oxidative cyclization reaction. Moderate yields (25\%-54\%) of 131 and 132 were obtained by refluxing dry toluene with $\operatorname{Pd}\left(\mathrm{PPh}_{3}\right)_{4}$ or $\mathrm{Pd}(\mathrm{dba})_{2}$ and $\mathrm{Ph}_{3} \mathrm{As}$ as catalysts. However, the copper(I)-catalyzed Sonogashira reactions between TBNDI and phenylacetylene or trimethylsilylacetylene 
produced a complex mixture and a small amount (4-8\%) of the desired products. ${ }^{73}$

2.7.3 Other designing strategies. As shown in Scheme 11, some other modifications of TBNDI, such as tetraamino-substitution, halogen-exchange, tetracyano-substitution and debromination, were also reported. SNAr substitution of TBNDI with benzophenone imine happened at $150-160{ }^{\circ} \mathrm{C}$ to afford 133 in 59\% yield, where an unexpected byproduct 135 was observed with the addition of a drop of water. Compound 135 could also be prepared by direct hydrolysis of 133 in DMSO with a drop of $\mathrm{HCl}^{74}$ Tetraamino-substituted compound 134 (TANDI) was synthesized through further hydrolysis of $\mathbf{1 3 3}$ under mild conditions in $42 \%$ yield. $^{75}$ As a rare type of NDAbased p-type semiconductor, compound $\mathbf{1 3 4}$ acted as a promising building block to construct more abundant $\pi$-conjugated OSC materials. ${ }^{76}$ The tetrafluoro-substituted NDI precursor (TFNDI) was synthesized from the TBNDI by halogen-exchange reactions under the conditions of CsF (approximately $101^{\circ} \mathrm{C}$ ) or KF and microwave heating $\left(200^{\circ} \mathrm{C}\right)$. TFNDI (136) was intrinsically unstable to water in refluxing dioxane during the reaction due to the strong electron-deficient core. However, the high reactivity of TFNDI ensured facile SNAr and subsequent oxidation to give 137 and $138 .^{77}$ Thus, more core-functionalizations based on the TFNDI precursor need to be exploited for various $\pi$-expanded OSCs. Stepwise cyanation of TBNDI was carried out to produce expectant mono-, di-, tri- and tetracyano-substituted derivatives. However, it was difficult to isolate tri- and tetracyano-substituted derivatives due to their high sensitivity to moisture. ${ }^{78}$ Mukhopadhyay et al. illustrated the first isolated tetracyano-substituted compound 140 and anionic state 139 with $\mathrm{PPh}_{4}{ }^{+}$ion-pairing, which were highly stable to air and chromatography. ${ }^{79}$ Some dehalogenation reactions of the above-mentioned annulated derivatives could also happen with the catalysis of $\mathrm{PbO}_{2}$ or $\mathrm{Pd} / \mathrm{C}$. Direct dehalogenation on TBNDI with Zn powder catalyst took place in the mixed solvent of isopropyl alcohol, HOAc and water. The regioselective reductive debromination afforded isomeric dibromides 141 and 142, which could also occur under microwave radiation. ${ }^{32}$ Further regioirregular polymerization of isomeric dibromide mixtures could be achieved through Stille coupling with organotin reagents and Pd-catalyst. ${ }^{21}$

\subsection{Polymerization from TBNDA}

Although hundreds of conjugated NDI-based polymers and oligomers have been investigated over the past few decades, most of them were attained from DBNDI and its derivatives. ${ }^{10}$ Syntheses of largely $\pi$-extended polymers from TBNDA or TBNDA derivatives are rare due to the inevitable steric constraints and complex active sites in the presence of four bromo-atoms. To address this issue, several synthetic methodologies have been exploited. As shown in Scheme 12, some promising polymeric precursors synthesized from TBNDI are listed. Compound 3 and its analogues with excellent solubility can proceed a condensation reaction with diamino monomers or benzenetetramine. Addition polymerization can be performed between compound $\mathbf{1 2}$ and styrene monomer. Notably,
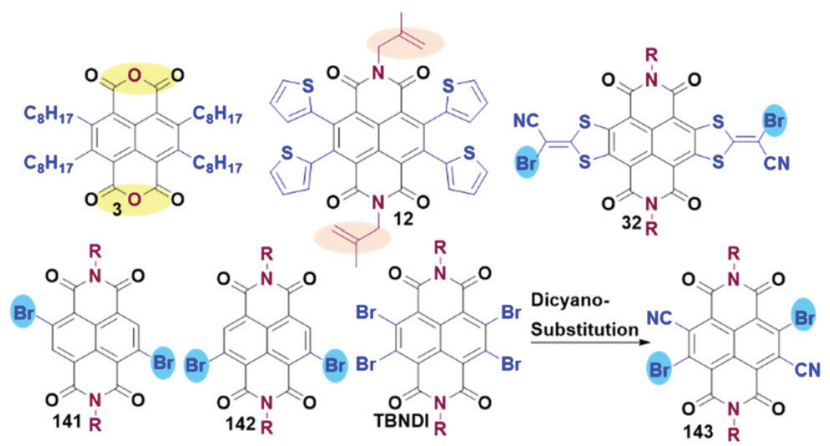

Scheme 12 Promising polymeric precursors synthesized from TBNDI.

the commonly-used method is to transform the tetrabromo- to dibromo-substitution NDI derivatives via SNAr with S-containing reagent and then bromination, regional dehalogenation or cyano-substitution, giving polymerization precursors $32,141,142$ and $143 .{ }^{80}$

\section{Optoelectronic properties and applications}

\subsection{OFET devices}

3.1.1 Molecular structure strategy. The development of high-performance ambient stable n-type OSCs for OFETs has made significant progress over the past few decades. ${ }^{2}$ Numerous functionalized NDA-based n-type OSCs have been designed for air-stable n-channel OFETs. ${ }^{80-85}$ As an essential branch of NDI derivatives, TBNDI emerged as a promising building block to construct abundant OSC materials for highperformance solution-processed OFET devices. ${ }^{86-90}$ It has been known that the unfunctionalized NDI exhibited high electron mobilities of $6.2 \mathrm{~cm}^{2} \mathrm{~V}^{-1} \mathrm{~s}^{-1}$ in an argon atmosphere, but low electron mobilities in air. ${ }^{80}$ Similarly, for some of the TBNDI derivatives (e.g., compound 60) cannot meet the requirement in OFETs because of their sensitivity to air and water caused by their high LUMO energies. To address this issue, the rational molecular design based on TBNDA is expected to obtain low-lying LUMO energies. A majority of molecular structure strategies and methodologies have been discussed in section 2 . To the best of our knowledge, by introducing strong electron-withdrawing groups (e.g., F, CN, fluoroalkyl groups) at the longitudinal $N$-position of the imide or lateral NDI core, stable n-type OSCs with low-lying LUMO energies can be achieved. In another way, the conjugated NDI framework incorporated electron-donating groups (e.g., carbazole, thiophene groups) can regulate the HOMO/LUMO energies to afford ambipolar and p-type semiconducting properties. $^{35,48,75,76}$ In general, the intrinsic energy level of TBNDI derivatives, particularly the LUMO energy level, is closely related to the air-stable electron injection and transport. It has been proven that the LUMO energy levels of the OSCs below $-4.0 \mathrm{eV}$, precisely ranging from -4.3 to $-4.4 \mathrm{eV}$, 
Table 4 Optical bandgaps $\left(E_{\mathrm{g}}\right)$, energy levels, and OFET performance of different TBNDI derivatives

\begin{tabular}{|c|c|c|c|c|c|c|c|}
\hline Compound & $\begin{array}{l}E_{\mathrm{g}} \\
(\mathrm{eV})\end{array}$ & $\begin{array}{l}\text { HOMO } \\
(\mathrm{eV})\end{array}$ & $\begin{array}{l}\text { LUMO } \\
(\mathrm{eV})\end{array}$ & $\begin{array}{l}\text { Deposition \& treatment } \\
\text { process }\end{array}$ & $\begin{array}{l}\mu_{\mathrm{e}} / \mu_{\mathrm{h}} \\
\left(\mathrm{cm}^{2} \mathrm{~V}^{-1} \mathrm{~s}^{-1}\right)\end{array}$ & $\begin{array}{l}\text { Measurement } \\
\text { conditions }\end{array}$ & Ref. \\
\hline 17 (R = 2-decyl-tetracosyl) & 2.10 & - & -4.30 & Spin-coating \& annealing $180^{\circ} \mathrm{C}$ & $0.15 /-$ & In air & 41 \\
\hline 17 (R = 2-octyl-dodecyl) & 2.00 & - & -4.30 & Spin-coating \& annealing $180^{\circ} \mathrm{C}$ & $0.51 /-$ & In air & 41 \\
\hline 17 (R = 2-octyl-dodecyl) & 2.00 & - & -4.30 & Spin-coating \& annealing & $3.5 /-$ & In $\mathrm{N}_{2}$ or air & 88 \\
\hline 17 (R = 2-octyl-dodecyl) & 2.00 & - & -4.30 & Spin-coating & $1.2 /-$ & In air & 89 \\
\hline 17 (R = 2-octyl-dodecyl) & 2.00 & - & -4.30 & Dip-coating & $0.005 /-$ & In air & 90 \\
\hline 19 (R = 2-octyl-dodecyl) & 2.50 & -6.60 & -4.10 & Spin-coating \& annealing $120^{\circ} \mathrm{C}$ & $0.0014 /-$ & In air & 44 \\
\hline 23 & 1.73 & -5.66 & -3.93 & Spin-coating \& annealing $120^{\circ} \mathrm{C}$ & $0.0026 / 0.0029$ & In $\mathrm{N}_{2}$ or air & 46 \\
\hline 24 & 1.82 & -5.73 & -3.91 & Spin-coating \& annealing $140^{\circ} \mathrm{C}$ & $0.047 / 0.016$ & In $\mathrm{N}_{2}$ or air & 46 \\
\hline 25 & 1.51 & -6.17 & -4.02 & Spin-coating \& annealing $180^{\circ} \mathrm{C}$ & $0.05 /-$ & In air & 47 \\
\hline $26(\mathrm{R}=$ butyl $)$ & 0.80 & -5.1 & -4.30 & Spin-coating \& annealing $160^{\circ} \mathrm{C}$ & $-/ 0.31$ & In air & 48 \\
\hline 27 & 2.00 & -5.80 & -3.80 & Spin-coating & $0.027 /-$ & In air & 44 \\
\hline $28(\mathrm{R}=$ hexyl $)$ & 0.9 & -5.00 & -4.10 & Spin-coating \& annealing $160^{\circ} \mathrm{C}$ & $0.003 / 0.03$ & In air & 48 \\
\hline 29 (R = 2-octyl-dodecyl) & 2.40 & -6.80 & -4.40 & Spin-coating \& annealing $80^{\circ} \mathrm{C}$ & $0.018 /-$ & In air & 44 \\
\hline 30 & 1.66 & -5.88 & -4.22 & Spin-coating \& annealing $120^{\circ} \mathrm{C}$ & $0.22 /-$ & In $\mathrm{N}_{2}$ or air & 46 \\
\hline 31 & 1.66 & -5.89 & -4.23 & Spin-coating \& annealing $120^{\circ} \mathrm{C}$ & $0.05 /-$ & In $\mathrm{N}_{2}$ or air & 46 \\
\hline 33 & 1.49 & -5.94 & -4.23 & Spin-coaling \& annealing $120^{\circ} \mathrm{C}$ & $0.37 /-$ & In air & 49 \\
\hline 34 & 1.56 & -5.93 & -4.15 & Spin-coating \& annealing $160^{\circ} \mathrm{C}$ & $0.24 /-$ & In air & 49 \\
\hline 35 & 1.70 & -5.56 & -4.17 & Spin-coating \& annealing $120^{\circ} \mathrm{C}$ & $0.10 /-$ & In air & 49 \\
\hline 36 & 1.52 & -5.82 & -4.25 & Spin-coating \& annealing $120^{\circ} \mathrm{C}$ & $0.38 /-$ & In air & 43 \\
\hline 37 & 1.15 & -5.63 & -4.29 & Spin-coating \& annealing $120^{\circ} \mathrm{C}$ & $0.08 /-$ & In air & 43 \\
\hline 40 & 1.61 & -6.24 & -3.93 & Spin-coating \& annealing $170^{\circ} \mathrm{C}$ & $0.04 /-$ & In air & 47 \\
\hline 55 & 1.99 & -6.29 & -4.30 & Spin-coating \& annealing 150 OC & $0.002 /-$ & In $\mathrm{N}_{2}$ & 53 \\
\hline 59 & 2.01 & -6.54 & -4.53 & Spin-coating \& annealing $150{ }^{\circ} \mathrm{C}$ & $0.005 /-$ & In $\mathrm{N}_{2}$ & 53 \\
\hline 62 & 2.38 & -6.83 & -4.45 & Dip-coating & $0.03 /-$ & In air & 54 \\
\hline 73 & 1.42 & -4.98 & -3.56 & Spin-coating \& annealing $140^{\circ} \mathrm{C}$ & $-/ 0.02$ & In air & 57 \\
\hline 74/76 & 2.38 & -6.37 & -3.99 & Spin-coating \& annealing $160^{\circ} \mathrm{C}$ & $0.15 /-$ & In air & 58 \\
\hline $75 / 77$ & 2.24 & -6.22 & -3.98 & Spin-coating \& annealing $160^{\circ} \mathrm{C}$ & $0.071 /-$ & In air & 58 \\
\hline $90(\mathrm{R}=2$-octyl-decyl $)$ & 1.15 & -5.56 & -4.41 & Single-crystal & $2.17 / 0.30$ & In air & 63 \\
\hline 98 & 1.7 & - & -4.25 & Spin-coating \& annealing & $0.1 /-$ & In air & 40 \\
\hline 108 & - & -5.08 & -4.05 & Spin-coating & $0.02 / 0.01$ & In air & 65 \\
\hline 109 & - & 5.10 & -4.49 & Spin-coating & $0.0002 /-$ & In air & 65 \\
\hline 110 & 1.09 & -5.27 & -4.35 & Spin-coating \& annealing & $0.007 /-$ & In air & 66 \\
\hline 112 & 0.99 & -5.30 & -4.72 & Spin-coating \& annealing & $0.96 /-$ & In air & 66 \\
\hline 125 & 1.64 & -5.62 & -4.10 & Spin-coating \& annealing $90^{\circ} \mathrm{C}$ & $0.00074 / 0.0009$ & In air & 72 \\
\hline 134 & - & -4.87 & -3.18 & Spin-coating \& annealing $80^{\circ} \mathrm{C}$ & $-/ 0.000082$ & In air & 75 \\
\hline 134/135 (mixed molar ratio $1: 3$ ) & - & - & - & Spin-coating \& annealing $80^{\circ} \mathrm{C}$ & $0.01 / 0.001$ & In $\mathrm{N}_{2}$ or air & 75 \\
\hline $136(\mathrm{R}=$ hexyl $)$ & - & -7.03 & -3.85 & Vacuum deposition at $100^{\circ} \mathrm{C}$ & $0.02 /-$ & In air & 77 \\
\hline
\end{tabular}

are most suitable for the air-stable OFET devices. ${ }^{86,87}$ Systemic molecular designs have been performed to figure out the relationship between the D/A structures and the corresponding optoelectronic properties. Optical bandgaps, energy levels, and OFET characteristics $\left(\mu_{\mathrm{e}} / \mu_{\mathrm{h}}\right.$, electron mobility/hole mobility) are summarized in Table 4 . In this principle, more and more TBNDI-based compounds with low-lying LUMO and stable architectures have been designed and synthesized for high-performance OFETs.

3.1.2 n-Type TBNDI-based OSCs. A majority of n-type small molecules synthesized from TBNDA have been developed as superior contenders for OFET devices. For instance, Zhu and Gao et al. have reported various malonitrile-based NDI derivatives with different substituted side chains and branching positions, such as compounds 17, 33, 98, etc. This strategy not only improves intermolecular $\pi-\pi$ stacking, but also lowers the LUMO energies. Fig. 1 displays the HOMO and LUMO of the representative compounds $17,110,112,108$, and 109. It can be seen that good electron delocalization states could form by rational molecular design. Thus, air-stable charge carrier transport behaviors could be successfully achieved. To further investigate the relationship between structural factors and charge transport properties through the measurement of the OFET performance, they found that the length and branching positions of alkyl chains effectively affected the intermolecular packing, crystallinity, and thin-film morphology, which may lead to variable semiconducting properties. For the OFETs based on bilateral S-containing annulation 17, the improved lamellar ordering and crystallinity with the increasing annealing temperature were responsible for the superior electron mobility of $0.51 \mathrm{~cm}^{2} \mathrm{~V}^{-1}$ $\mathrm{s}^{-1}$ and high current on/off ratios of $10^{5}-10^{7} .^{41}$ The derivative of compound 17 with three-branched $N$-alkyl substituents of $\mathrm{C}_{11,6}(\mathrm{R}$ = 2-octyl-dodecyl) exhibited an unprecedented electron mobility of $3.50 \mathrm{~cm}^{2} \mathrm{~V}^{-1} \mathrm{~s}^{-1} \cdot{ }^{80,88}$ Some other TBNDI-based small molecules for n-type or ambipolar transport behavior are summarized in Table 4. For n-type pure electron polymers, most of their bottom gate OFET devices generally exhibited mobilities $<0.3 \mathrm{~cm}^{2} \mathrm{~V}^{-1} \mathrm{~s}^{-1}$. However, Gao et al. reported that compound 36 showed unipolar n-channel charge transport with an electron mobility of $0.38 \mathrm{~cm}^{2}$ $\mathrm{V}^{-1} \mathrm{~s}^{-1}$, an on/off current ratio of $10^{6}$ and a threshold voltage of $-4.32 \mathrm{~V}$, which was superior to most of the n-type polymers. ${ }^{43}$

3.1.3 p-Type and ambipolar TBNDI-based OSCs. Till now, only a few cases of core-extended NDI derivatives have been 


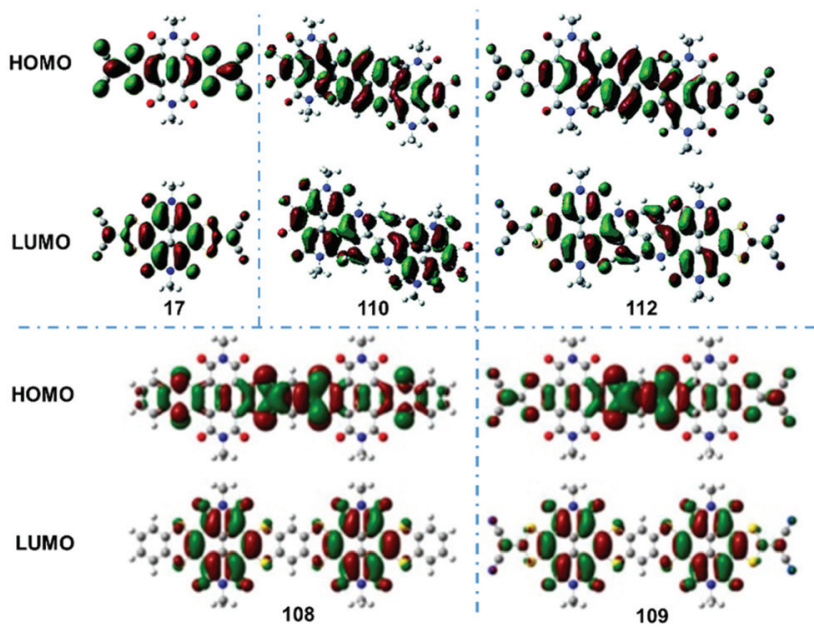

Fig. 1 HOMO and LUMO orbitals of the representative compounds 17, 110, 112, 108, 109. Reproduced with permission. ${ }^{41}$ Copyright 2010 American Chemical Society. Reproduced with permission. ${ }^{66}$ Copyright 2016, Royal Society of Chemistry. Reproduced with permission. ${ }^{65}$ Copyright 2019, American Chemical Society.

reported to exhibit p-type (hole) transport properties. In 2011, Würthner $e t$ al. reported the first p-channel transport of corecarbazole-annulated NDI with branched alkyl chains. The HOMO and LUMO levels were calculated to be -3.73 and $-5.91 \mathrm{eV}$. By measuring the output and transfer characteristics of this compound in ambient air on $\mathrm{Si} / \mathrm{SiO}_{2}(100 \mathrm{~nm}) / \mathrm{AlO}_{x}$ $(8 \mathrm{~nm}) / \mathrm{SAM}$ (a fluoroalkyl phosphonic acid (FC18-PA) selfassembled monolayer), a p-type behavior was observed with a field-effect mobility of $0.56 \mathrm{~cm}^{2} \mathrm{~V}^{-1} \mathrm{~s}^{-1}$, vanishingly small electron mobility and an on/off current ratio of $10^{6}$. Further investigation manifested that ambipolar properties could be obtained with the hole mobility of $0.03 \mathrm{~cm}^{2} \mathrm{~V}^{-1} \mathrm{~s}^{-1}$ and an electron mobility of $0.02 \mathrm{~cm}^{2} \mathrm{~V}^{-1} \mathrm{~s}^{-1}$ on a different dielectric architecture $\left(\mathrm{Si} / \mathrm{Al}(20 \mathrm{~nm}) / \mathrm{AlO}_{x}(3.6 \mathrm{~nm}) / \mathrm{SAM}\right)$ with a different SAM layer (FC18-PA or an alkyl phosphonic acid (HC14-PA) SAM). The outstanding p-type transport behavior could be explained by its structural features, which was in favor of intermolecular HOMO overlapping by the stacking modes of the extended $\pi$-core. ${ }^{76 a}$ Inspired by this concept, ambipolar OSC compound 125 was designed and synthesized. It was found that compound 125 based OFETs exhibited n-type behavior under a $\mathrm{N}_{2}$ atmosphere with an average electron mobility of $4.83 \times 10^{-3} \mathrm{~cm}^{2} \mathrm{~V}^{-1} \mathrm{~s}^{-1}$ and the on/off current ratio of $5 \times 10^{5}$. However, the device exhibited typical ambipolar feature in air with a mobility of $7.4 \times 10^{-4}$ for electrons and $9.0 \times 10^{-4} \mathrm{~cm}^{2}$ $\mathrm{V}^{-1} \mathrm{~s}^{-1}$ for holes and the on/off current ratio of $10^{3}-10^{4} .^{72}$ Zhang et al. reported D/A compound 26 as a p-type semiconductor and compound 28 as an ambipolar semiconductor. Compound 26 showed the best hole mobility of $0.31 \mathrm{~cm}^{2} \mathrm{~V}^{-1}$ $\mathrm{s}^{-1}$ and on/off current ratio of $10^{4}$ after annealing at $160{ }^{\circ} \mathrm{C}$, whereas compound 28 exhibited the respective mobility of 0.03 and $0.003 \mathrm{~cm}^{2} \mathrm{~V}^{-1} \mathrm{~s}^{-1}$ for holes and electrons in air after annealing. ${ }^{48}$ Moreover, compound TANDI (134) behaves as a p-type semiconductor with a high HOMO energy $(4.87 \mathrm{eV})$ and a low hole mobility of $8.2 \times 10^{-5} \mathrm{~cm}^{2} \mathrm{~V}^{-1} \mathrm{~s}^{-1}$. In order to enhance the performance, an efficient strategy was proposed that blending compounds $\mathbf{1 3 4}$ and $\mathbf{1 3 5}$ to form D-A complexes could improve ambipolar charge-transport behavior. The results showed that when the mixed molar ratio reached $1: 3$, the best performance was achieved with individual electron and hole mobilities of 0.01 and $0.001 \mathrm{~cm}^{2} \mathrm{~V}^{-1} \mathrm{~s}^{-1}$,respectively. ${ }^{72}$ Further studies on D-A complexes and novel p-type or ambipolar air-stable OSCs are needed to develop TBNDI-based high-performance OFET devices.

3.1.4 OFET device manufacture. Besides the molecular structural design, device fabrication conditions (spin-coating or vacuum evaporation), the treatment process (annealing time and temperature), and the thickness and morphology of ultrathin films and electrodes all act as significant factors for the OFET performance. Pei et al. designed and synthesized the bis-TBNDI derivatives 110 and 112. It was noticed that $\mathbf{1 1 2}$ revealed high sensitivity to the annealing temperature. Its electron mobility can be modified from 0.12 to $1.0 \mathrm{~cm}^{2} \mathrm{~V}^{-1} \mathrm{~s}^{-1}$ with the increased annealing temperature from 100 to $250{ }^{\circ} \mathrm{C}$. The desired performances with low threshold voltages of -5 to $0 \mathrm{~V}$ can be obtained at the optimized annealing temperature. ${ }^{66}$ Gao et al. found that the OFET properties of a compound 17 derivative $(\mathrm{R}=2$-octyl-dodecyl $)$ can be affected by the working electrodes. A lower contact resistance can be generated between the Ag-source/drain electrodes and the Ag-source/ drain electrodes affording a higher electron mobility of $0.51 \mathrm{~cm}^{2} \mathrm{~V}^{-1} \mathrm{~s}^{-1}$, while Au-contact devices exhibited satisfactory operating stability under ambient conditions. ${ }^{41}$ Furthermore, precise thin-film thickness was controlled by depositing OSC materials on OTS-modified $\mathrm{SiO}_{2}$ surfaces. Mono- and bi-layer thin-films of compound $\mathbf{1 7}$ derivatives were prepared and applied in OFET devices, which showed that the defined few-layer films demonstrated a decent electron mobility up to $1.2 \mathrm{~cm}^{2} \mathrm{~V}^{-1} \mathrm{~s}^{-1} .{ }^{89}$ Additionally, morphology engineering and molecular ordering also play a crucial role in influencing their OFET performances. ${ }^{91}$ For example, the ultrathin film of compound 17 ( $\mathrm{R}=2$-octyl-dodecyl) fabricated via the dip-coating method exhibited a much lower electron mobility of $0.005 \mathrm{~cm}^{2} \mathrm{~V}^{-1} \mathrm{~s}^{-1}$ than the reported value, which was partially attributed to the lower molecular ordering. ${ }^{90}$ Thus, research on efficient film-preparation and morphology-controlling techniques proves to be an appealing topic for OFET applications.

\subsection{Fluoride sensor}

The sensing and recognition of ions have been studied for several decades in supramolecular and biological chemistry based on several driving forces (i.e., classic hydrogen-bonding and electrostatic interactions)..$^{92}$ Notably, the selectivity and sensitivity are two dominant criteria to evaluate anion chemosensors. ${ }^{93-97}$ Core-substituted TBNDI derivatives are considered as ideal materials for sensors due to their excellent spectroscopic and electrochemical properties. In Fig. 2a, the unilateral $\mathbf{N}$-heterocyclic compound $\mathbf{4 2}$ with a functionalized 


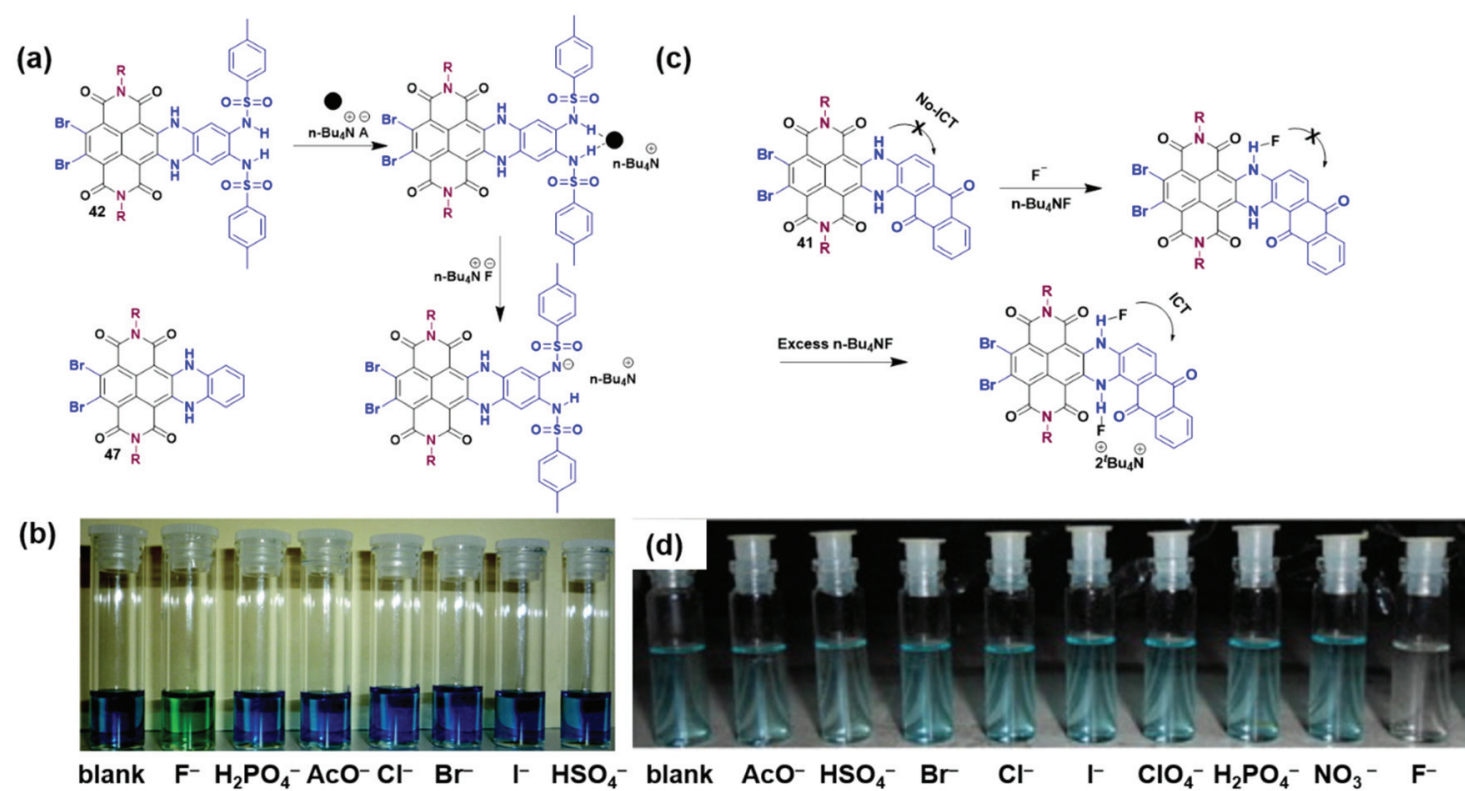

Fig. 2 (a) Anion sensor structure illustrating where the anion binds. (b) Sensing capability with color changes upon addition of 3 eq. of anions ( $\mathrm{F}^{-}$,

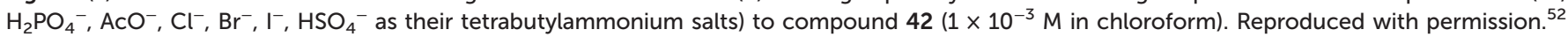
Copyright 2009, American Chemical Society. (c) Anion sensor structure illustrating the recognition of fluoride via an ICT effect. (d) Color changes upon the addition of 5 eq. of anions (as their tetrabutylammonium salts) to compound $41\left(1 \times 10^{-5} \mathrm{M}\right.$ in chloroform). Reproduced with permission. ${ }^{51}$ Copyright 2015, Elsevier.

disulfonamide group was designed as a sensor receptor. When different tetrabutylammonium salts (3 eq.) containing different anions (i.e., $\mathrm{F}^{-}, \mathrm{H}_{2} \mathrm{PO}_{4}{ }^{-}, \mathrm{AcO}^{-}, \mathrm{Cl}^{-}, \mathrm{Br}^{-}, \mathrm{I}^{-}$, or $\mathrm{HSO}_{4}{ }^{-}$) were added to the $\mathrm{CHCl}_{3}$ solution of 42, respectively (Fig. 2b), compound 42 was found to display a high selectivity to fluoride, demonstrating the viable application in colorimetric $\mathrm{F}^{-}$ sensors. To explore the detection mechanism, UV-Vis, fluorescence, electrochemistry, ${ }^{1} \mathrm{H}$ NMR spectroscopy and DFT calculations were performed to monitor and quantify the fluoride-induced spectral changes with different anion proportions and solvents. In addition, compound $\mathbf{4 7}$ was employed as a contrast test, where no change was observed after adding the same anions, manifesting that the fluoride detection had no connection with the protons in the $\mathrm{NH}$ groups or anion- $\pi$ interactions. After analyzing these results, a deprotonation mechanism was proposed in Fig. 2a. The addition of the first $\mathrm{F}^{-}$ion realized the formation of an $\mathrm{H}$-bonded complex, followed by the deprotonation of the bis-sulfonamide with the second $\mathrm{F}^{-}$ion, leading to the creation of $\mathrm{HF}_{2}{ }^{-}$. The deprotonation process could be reversed by adding polar protic solvents, such as $\mathrm{CH}_{3} \mathrm{OH}$ or EtOH. ${ }^{52}$ Compound $\mathbf{4 1}$ was also reported to possess high selectivity and sensitivity for fluoride over other anions (i.e., $\mathrm{AcO}^{-}, \mathrm{HSO}_{4}^{-}, \mathrm{Br}^{-}, \mathrm{Cl}^{-}, \mathrm{I}^{-}, \mathrm{ClO}_{4}{ }^{-}, \mathrm{H}_{2} \mathrm{PO}_{4}{ }^{-}$, and $\mathrm{NO}_{3}{ }^{-}$), as shown in Fig. 2d. The possible detection mechanism is shown in Fig. 2c. It was speculated that the H-bonding interaction and subsequent deprotonation happened between the $\mathrm{NH}$ moiety and the firstly-added $\mathrm{F}^{-}$anion. The interaction between excess $\mathrm{F}^{-}$anions and the remaining $\mathrm{NH}$ moiety induced the delocalization of negative charges over the mole- cule, which created an intramolecular charge transfer (ICT) effect. ${ }^{51}$ Furthermore, Zhu et al. proposed a new detection mechanism based on compound 20 that the observed paramagnetic radical anions and the ICT process from $\mathrm{F}^{-}$to NDI core dominated the $\mathrm{F}^{-}$anion sensing. However, the radicalanion-ICT mechanism remains unintelligible and requires intensive research. ${ }^{31}$ The sensing behaviors of $\mathbf{1 3 3}$ in organic and aqueous solutions has already been investigated. Compound 133 could act as an efficient $\mathrm{F}^{-}$anion sensor in the organic solution due to the existence of $\mathrm{NH}$ fragments. ${ }^{74}$ From the above results, it can be concluded that $\mathrm{N}$-heterocyclic TBNDI derivatives are well suitable for anion sensing application. More modified TBNDI-based compounds should be considered as promising candidates for the detection of different anions or metal cations (i.e., $\mathrm{F}^{-}, \mathrm{Cl}^{-}, \mathrm{I}^{-}, \mathrm{Hg}^{+}, \mathrm{Cu}^{2+}$ ) and other applications (e.g., anion transport and purification). More importantly, the specific mechanisms of ion detection are required to be figured out in the future.

\subsection{Molecular self-assembly}

The controllable micro- and nano-scale assemblies of organic conjugated materials (including NDI analogues) via self-assembly have been widely investigated in sensors, catalysis and electronic devices. ${ }^{98-102}$ For example, some non-halogenated NDA derivatives presented intriguing self-assembly behaviors due to their abundant crystal form and numerous polymorphic transitions. ${ }^{103,104}$ For example, dibromide-substituted NDA derivatives with rational molecular modification could also achieve diverse self-assemblies by strategically tailoring non- 


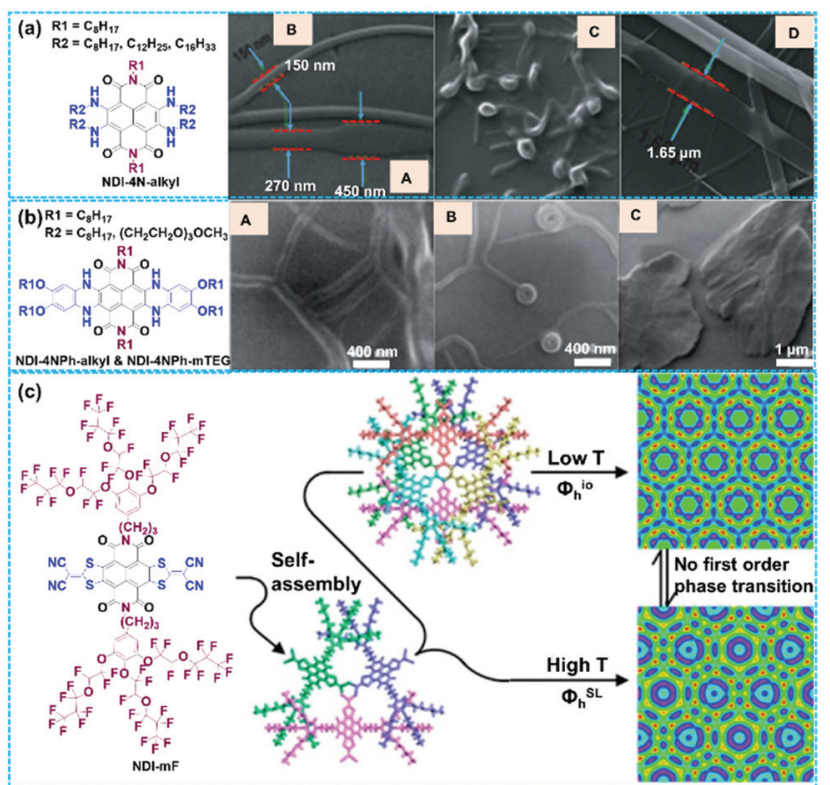

Fig. 3 (a) Molecular structures of NDI-4N-alkyl and the respective SEM micrographs $(A-D)$ revealing the formation of supramolecular nanostructures. Reproduced with permission. ${ }^{109}$ Copyright 2014, Wiley-VCH. (b) Molecular structures of NDI-4NPh-alkyl and NDI-4NPh-mTEG and the respective SEM micrographs $(A-C)$ revealing the formation of supramolecular nanostructures. Reproduced with permission. ${ }^{110}$ Copyright 2013, Royal Society of Chemistry. (c) Molecular structure of a dendronized TBNDI derivative functionalized with the semifluorinated group (NDI-mF), and the electron density map overlaid with the schematic representation of the column structure for the low-temperature $\Phi h^{\text {io }}$ phase and the high-temperature $\Phi h^{\mathrm{SL}}$. Reproduced with permission. ${ }^{112}$ Copyright 2014, American Chemical Society.

covalent interactions such as H-bonds, hydrophobic interactions, intermolecular $\pi-\pi$ stacking, ionic and electrostatic interactions, van der Waals interactions, etc. Thus, welldesigned functionalization at the longitudinal imide nitrogen atoms and lateral halogen atoms of NDI and DBNDI provides an efficient strategy to fulfill diverse self-assembly methodologies. ${ }^{105-108}$ However, the research on functionalized TBNDIs is inadequate to clearly understand the self-assembly mechanism affected by lateral and longitudinal substituents. Tetra-alkylamino-substituted compounds NDI-4N-alkyl with different alkyl chain lengths $\left(\mathrm{C}_{8} \mathrm{H}_{17}, \mathrm{C}_{12} \mathrm{H}_{25}\right.$, and $\left.\mathrm{C}_{16} \mathrm{H}_{33}\right)$ were synthesized to investigate self-assembly behavior. As shown in Fig. 3a, a variety of well-defined nanoarchitectures, such as nanorods, vesicular, belts, twisted ribbons and donut-like morphologies, were produced via solvophobic control, which was mainly attributed to the packing mode of hydrophobic alkyl chains and the $\pi-\pi$ stacking interaction of the TBNDI core. ${ }^{109}$ Examples of self-assembly of the bilateral alkyl-annulated TBNDIs are shown in Fig. $3 \mathrm{~b}$. The bilateral core-annulation TBNDI derivatives with long alkyl chains (NDI-4NPh-alkyl) or methoxytriethyleneglycol (NDI-4NPh-mTEG) could form various controllable nanostructures (including tubular nanofiber, donut-, needle-, flake- and leaf-like microstructures) by solvophobic control. ${ }^{110}$ Similarly, the unilateral alkyl-annu- lated derivatives self-assembled into wormlike nanostructures, while mTEG-annulated derivatives self-assembled into multilamellar vesicular aggregates with a defined diameter. ${ }^{50,111} \mathrm{~A}$ series of compound $\mathbf{1 7}$ derivatives with chiral racemic semifluorinated groups and $\mathrm{AB}_{3}$-type minidendrons ( $m=1,2$ or 3 ) were reported for supramolecular assembly. The TBNDI derivatives with a semifluorinated group and dendron alkyl units $(m$ $=2$ ) self-organized in lamellar crystals via a thermodynamically controlled process. The dendronized TBNDI derivative $(m=1)$ self-organized in lamellar crystals via a kinetically controlled process. Besides, the dendronized TBNDI derivative with $m=3$ thermodynamically self-organized in a columnar hexagonal periodic array with an unprecedentedly complex and ordered column, that is, a columnar hexagonal lattice $\left(\Phi \mathrm{h}^{\mathrm{io}}\right)$ at low temperatures and a superlattice at high temperatures $\left(\Phi \mathrm{h}^{\mathrm{SL}}\right)$ in Fig. 3c. Although thermodynamically controlled processes dominated both assemblies and periodic arrays of TBNDI derivatives with $m=3$, no first-order phase transition could be observed. ${ }^{112}$ Therefore, supramolecular nanoarchitectures and rigorous methodologies of TBNDI derivatives should be elaborated for a better understanding of self-assembly behavior.

\subsection{Other applications: gas sensors, self-colored} nanoparticles, J-aggregation behaviors and stable radical anions

The elaboration of TBNDI-based OSCs has attracted tremendous interest in their applications in other fields, including gas sensors, the formation of radical anions, self-colored nanoparticles, and J-aggregation behaviors. As shown in Fig. 4a, a specific and reproducible OFET-based gas sensor was developed with the electron-deficient TBNDI-derivative (compound 17) as the receptor element. The operation of this device involved the process of gas adsorption, reacting with the active layer, and then the change in charge transport. The chemical reactivity of the receptor molecules plays a vital role in sensing performance. Specifically, the non-covalent interactions or chemical reactions between the analytes and active layer could affect the carrier density and/or charge transport in the conduction channel, which can be monitored by the electrical signal on OFET devices. As a result, specific and reproducible gas-phase $\left(\mathrm{HCl}, \mathrm{NO}_{2}\right.$, and $\left.\mathrm{NH}_{3}\right)$ detection has been realized. ${ }^{113}$ Fig. $4 \mathrm{~b}$ displays the fabrication of self-colored nanoparticles by mini-emulsion copolymerization between styrene monomer and TBDA derivatives (compounds 12 and 15). The as-obtained self-colored nanoparticles could act as the protein solid for application in diagnostic fields, e.g., latex agglutination assay or lateral flow immunochromatographic assay. ${ }^{20}$ Furthermore, some other essential properties of TBNDI-based materials have been explored, such as intermolecular redox doping, nearinfrared J-aggregation behavior, ambient stability of the radical anion, and so on. The intensive comprehension of the energetic prerequisites for electron transport may guide the rational design of TBNDI derivatives with the controllable conductivity through self-doping, which could be applied in organic thermoelectric devices. ${ }^{114}$ In Fig. 4c, well-defined nearinfrared J-aggregation behaviors of 105 and 106 elucidated 

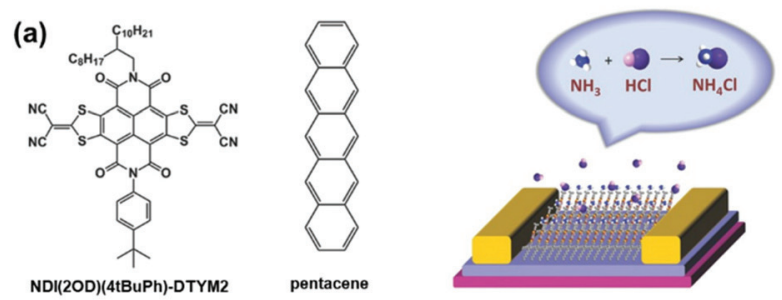

(c)
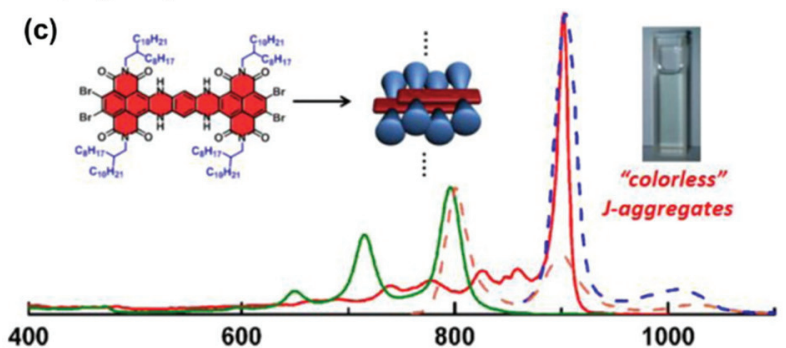

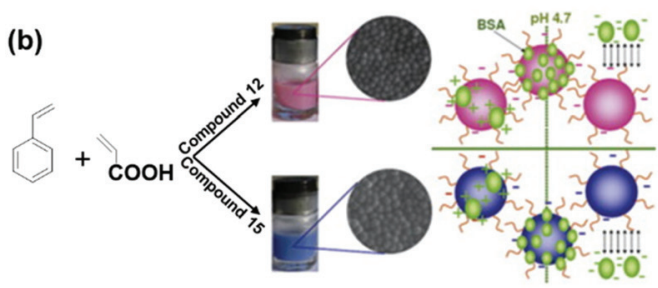

(d)
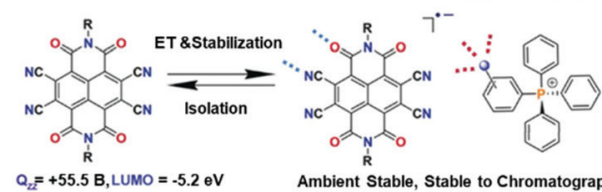

$Q_{2 L}=+55.5 \mathrm{~B}$, LUMO $=-5.2 \mathrm{eV}$

Ambient Stable, Stable to Chromatography

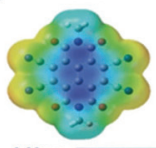

0.06 a.u.

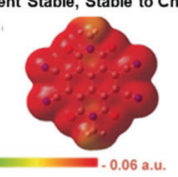

Fig. 4 (a) Molecular structures of the n-type TBNDI derivative and p-type pentacene, and a schematic diagram of the device structure and sensing mechanism. Reproduced with permission. ${ }^{113}$ Copyright 2014, Wiley-VCH. (b) Preparation of self-colored nanoparticles containing TBNDI derivatives. Reproduced with permission. ${ }^{20}$ Copyright 2011, Elsevier. (c) Schematic representation of the proposed packing motifs of the studied bis-TBNDIs and absorption (solid) and emission (dash) spectra $\left(1.0 \times 10^{-6} \mathrm{M}\right)$ in $\mathrm{CHCl}_{3}$ (green and pink) and $n$-hexane (red and blue). Reproduced with permission. ${ }^{64}$ Copyright 2014, American Chemical Society. (d) Structure of compound 140 and its radical anion compound 139 stabilized by supramolecular interactions. ESP (electrostatic surface potential) maps of compound 140 (left) and its radical anion (right). ESP contours are color-coded from red (electron-rich) to blue (electron-deficient). Reproduced with permission. ${ }^{78}$ Copyright 2018 , Wiley-VCH.

specific queries, appealing to more applications such as organic photovoltaics (OPVs), heat-blocking coating, optical filter, information-security display, and so on. ${ }^{66}$ In Fig. 4 d, tetracyano-TBNDI and its radical anion stabilized by supramolecular interactions were first isolated as planar radical ion systems. Anion- $\pi$ interactions, and magnetic and conductive properties of TBNDI $\pi$-systems are attractive for anion recognition and transport, ionic assembly in the radical anions, medicinal and materials chemistry, and switchable electronics. ${ }^{78}$

\subsection{Promising applications: thermoelectric devices, and resistive memory devices}

Although these TBNDI derivatives have the features of a $\pi$-conjugated framework, an impressive LUMO energy level and a good ability, these OSC materials still remain in an inadequate development stage. In order to exploit more applications, here, some of the promising applications are proposed, such as thermoelectric and resistive memory fields. As we have known, organic thermoelectric materials (OTEs) have achieved considerable progress in the past few decades, which realize the heat-electricity conversion from solar energy or waste heat. Most of the OSC-based thermoelectric (TE) devices require optimal architectures of $\mathrm{n}$ - and $\mathrm{p}$-type OSCs and dopants to control the charge carrier for high electrical conductivities and Seebeck coefficients. ${ }^{115}$ Some NDI-based polymer fused thiophene or thiazole groups have been successfully applied to TE devices with high electrical conductivity and stable thermoelectric response. ${ }^{116}$ However, till now, most of the TBNDI derivatives have not received adequate attention in TE application. Few examples of compound 17 derivatives have been reported. ${ }^{117}$ Analogously, organic resistive memory devices (ORMDs) have emerged as a potential contender to realize ultrahigh-density data storage in the near future. A variety of functional D-A conjugated OSC materials have been developed. ${ }^{6}$ However, the long-term stability of the fabricated device remains as one of the obstacles in the way to practical application. Thus, we expect these TBNDI derivatives to be applied in ORMDs for anticipated performance.

\section{Conclusions}

In this review, we focus on the recent progress in the synthetic strategies of TBNDA derivatives and their applications. The key synthetic strategies and rational molecular design are elucidated in a precise and complete way, where esterification, longitudinal imidization and direct core-functionalization of TBNDA are the primary approaches to produce different synthons (e.g., TBNDI, TBNDE, TBNDF). In particular, TBNDI derived from TBNDA has been recognized as the most versatile intermediate for further annulated or non-annulated modification on the lateral TBNDI core. The electron-deficient TBNDI with tetrabromo-substituents ensures efficient stepwise SNAr reaction with electron-donating $\mathrm{S}-, \mathrm{N}-$, O-containing nucleophiles. Moreover, polyacene and heterocycle-fused polyacene analogues are feasible to be prepared through the SNAr and metal-catalyzed cross-couplings reactions. In particular, dimers, oligomers and polymers are discussed to form a meticulous summary. Besides, many other reactions such as condensation, bromination, dehalogenation, halogen-exchange, oxidation and reduction could also be conducted in TBNDA/ 
TBNDI-based derivatives, where these materials could be classified as unilateral and bilateral annulation compounds, non-annulated tetra-substitution materials, and selective substitution products.

Motivated by the advantages of diverse molecular design, ambipolar nature, intensive absorption in the NIR region, outstanding $\pi$-acidity and low lying LUMO levels, etc., various potential applications are also presented. High-performance ambient-stable n-type, p-type and ambipolar TBNDI-based OFETs have been fabricated with the electron mobility ranging from $10^{-4}$ to $3.5 \mathrm{~cm}^{2} \mathrm{~V}^{-1} \mathrm{~s}^{-1}$ and the hole mobility ranging from $10^{-5}$ to $0.3 \mathrm{~cm}^{2} \mathrm{~V}^{-1} \mathrm{~s}^{-1}$. Systemic molecular designs have been discussed to figure out the relationship between the D/A structures and the corresponding optoelectronic properties. It has been found that stable n-type OSCs with low-lying LUMO energies can be readily fabricated by incorporating strong electron-withdrawing groups, whereas p-type OFET behavior could be obtained with the introduction of electron-donating groups at the longitudinal $N$-position of imide or the lateral NDI core. However, ambipolar OFET performance can be realized by adjusting the experimental conditions. On the other hand, the formation of proper D-A complexes by the blending method is also deemed as an efficient strategy to improve ambipolar charge-transport behavior. Furthermore, the device fabrication conditions, the treatment process, and the thickness and morphology of ultrathin films and electrodes have been taken into consideration. Some other applications, such as fluoride sensors with high selectivity and sensitivity, supramolecular self-assembly and polymorphic transitions, OFET-based gas sensors, stable radical anions, self-colored nanoparticles and NIR J-aggregation behaviors, are also investigated in depth.

However, a lot of challenges still remain in the way to develop TBNDA derivatives and expand their applications: (1) additional synthetic strategies and molecular design to construct more tailored TBNDA-based small molecules and polymers are highly desirable for diverse potential applications; (2) an intensive comprehension of structure-property relationships to obtain better electronic properties or self-assembly behaviors is urgent; and (3) although some of the TBNDI derivatives have been demonstrated to exhibit excellent electron mobility, there is still plenty of room left to explore the possibilities of the applications of these materials in a broad range of fields, such as catalysis, biomedicine, diagnosis, solar cells, OPVs, thermoelectric devices, resistive memory devices, and so on.

\section{Conflicts of interest}

The authors declare no conflict of interest.

\section{Acknowledgements}

Q. Z. acknowledges the financial support from AcRF Tier 1 (RG111/17, RG 2/17, RG 114/16, RG 113/18) and Tier 2
(MOE2017-T2-1-021 and MOE 2018-T2-1-070), Singapore. J. L. and H. L. acknowledge the financial support from the NSF of China (21878199, 21808149, 21476152), the NSF of the Jiangsu Higher Education Institutions of China (grant no. 17KJA4300151 and 19KJB150018), and the Priority Academic Program Development of Jiangsu Higher Education Institutions (PAPD). QZ also acknowledges the support from the 111 Project (D20015) and the State Key Laboratory of Supramolecular Structure and Materials, Jilin University (sklssm2020041).

\section{References}

1 (a) H. Bronstein, C.-B. Nielsen, B.-C. Schroeder and I. McCulloch, The Role of Chemical Design in the Performance of Organic Semiconductors, Nat. Rev. Chem., 2020, 4, 66-77; (b) A. Facchetti, $\pi$-Conjugated Polymers for Organic Electronics and Photovoltaic Cell Applications, Chem. Mater., 2011, 23, 733-758.

2 (a) C. Wang, H. Dong, L. Jiang and W. Hu, Organic Semiconductor Crystals, Chem. Soc. Rev., 2018, 47, 422500; (b) X. Zhan, A. Facchetti, S. Barlow, T.-J. Marks, M.-A. Ratner, M.-R. Wasielewski and S.-R. Marder, Rylene and Related Diimides for Organic Electronics, Adv. Mater., 2011, 23, 268-284; (c) Y. Huang, J. Xing, Q. Gong, L.-C. Chen, G. Liu, C. Yao, Z. Wang, H.-L. Zhang, Z. Chen and Q. Zhang, Reducing Aggregation Caused Quenching Effect Through Co-Assembly of PAH Chromophores and Molecular Barriers, Nat. Commun., 2019, 10, 169; (d) Y. Huang, Z. Wang, Z. Chen and Q. Zhang, Organic Cocrystals: Beyond Electrical Conductivities and FieldEffect Transistors (FETs), Angew. Chem., 2019, 58, 96969711.

3 (a) S.-E. Root, S. Savagatrup, A.-D. Printz, D. Rodriquez and D.-J. Lipomi, Mechanical Properties of Organic Semiconductors for Stretchable, Highly Flexible, and Mechanically Robust Electronics, Chem. Rev., 2017, 117, 6467-6499; (b) F. Würthner, C.-R. Saha-Möller, B. Fimmel, S. Ogi, P. Leowanawat and D. Schmidt, Perylene Bisimide Dye Assemblies as Archetype Functional Supramolecular Materials, Chem. Rev., 2015, 116, 962-1052.

4 (a) S. Riera-Galindo, F. Leonardi, R. Pfattner and M. MasTorrent, Organic Semiconductor/Polymer Blend Films for Organic Field-Effect Transistors, Adv. Mater. Technol., 2019, 4, 1900104; (b) V. Punitharasu, M.-F. Mele Kavungathodi and J. Nithyanandhan, Self-Assembly of Cis-Configured Squaraine Dyes at the $\mathrm{TiO}_{2}$-Dye Interface: Far-Red Active Dyes for Dye-Sensitized Solar Cells, ACS Appl. Mater. Interfaces, 2018, 10, 16541-16551.

5 (a) Z. Zhang and Q. Zhang, Recent Progress in WellDefined Higher Azaacenes $(\mathrm{n} \geq 6)$ : Synthesis, Molecular Packing, and Applications, Mater. Chem. Front., 2020, DOI: 10.1039/C9QM00656G; (b) W. Chen, F. Yu, Q. Xu, G. Zhou and Q. Zhang, Recent Progress in High Linearly Fused Polycyclic Conjugated Hydrocarbons (PCHs, n > 6) with 
Well-Defined Structures, Adv. Sci., 2020, 7, 1903766; (c) K. Wang, X. Cao, S. Wang, W. Zhao, J. Xu, Z. Wang and $\mathrm{H}$. $\mathrm{Wu}$, Interpenetrated and Polythreaded CoII-Organic Frameworks as a Supercapacitor Electrode Material with Ultrahigh Capacity and Excellent Energy Delivery Efficiency, ACS Appl. Mater. Interfaces, 2018, 10, 91049115; (d) J. Li, S. Chen, Z. Wang and Q. Zhang, PyreneFused Acenes and Azaacenes: Synthesis and Applications, Chem. Rec., 2016, 16, 1518-1530; (e) Z. Wang, P. Gu, G. Liu, H. Yao, Y. Wu, Y. Li, G. Rakesh, J. Zhu, H. Fu and Q. Zhang, A Large Pyrene-Fused N-Heteroacene: Fifteen Aromatic Six-Membered Rings Annulated in One Row, Chem. Commun., 2017, 53, 7772-7775; (f) P. Gu, Z. Wang, G. Liu, H. Yao, Z. Wang, Y. Li, J. Zhu, S. Li and Q. Zhang, Synthesis, Full Characterization, and Field Effect Transistor Behavior of a Stable Pyrene-Fused N-Heteroacene with Twelve Linearly Annulated SixMembered Rings, Chem. Mater., 2017, 29, 4172-4175.

6 (a) Y. Li, Q. Qian, X. Zhu, Y. Li, M. Zhang, J. Li, C. Ma, H. Li, J. Lu and Q. Zhang, Recent Advances in OrganicBased Materials for Resistive Memory Applications, InfoMat, 2020, DOI: 10.1002/inf2.12120C; (b) C. Zhang, Y. Li, Y. Zhou, Q. Zhang, H. Li and J. Lu, Deriving Highly Oriented Organic Nanofibers and Ternary Memory Performance via Salification-Induced Effects, Chem. Commun., 2018, 54, 10610-10613.

7 (a) J. Yang, M. Fang and Z. Li, Stimulus-Responsive Room Temperature Phosphorescence in Purely Organic Luminogens, InfoMat, 2020, 2, 791-806; (b) J. Liu, Z. Qin, H. Gao, H. Dong, J. Zhu and W. Hu, Vertical Organic Field-Effect Transistors, Adv. Funct. Mater., 2019, 29, 1808453; (c) G. Sonmez, H. Meng, Q. Zhang and F. Wudl, A Highly Stable, New Electrochromic Polymer: Poly(1, 4-bis(2-(3', 4'-ethylenedioxy) thienyl)-2-methoxy-5-2"-ethylhexyloxybenzene), Adv. Funct. Mater., 2003, 13, 726-730.

8 Y. Liu, Y. Yang, D. Shi, M. Xiao, L. Jiang, J. Tian, G. Zhang, Z. Liu, X. Zhang and D. Zhang, Photo-/ThermalResponsive Field-Effect Transistor upon Blending Polymeric Semiconductor with Hexaarylbiimidazole toward Photonically Programmable and Thermally Erasable Memory Device, Adv. Mater., 2019, 31, 1902576.

9 (a) F. Yu, W. Liu, B. Li, D. Tian, J. L. Zuo and Q. Zhang, Photostimulus-Responsive Large-Area Two-Dimensional Covalent Organic Framework Films, Angew. Chem., 2019, 58, 16101-16104; (b) X. Zhan, Z. Chen and Q. Zhang, Recent Progress in Two-Dimensional COFs for EnergyRelated Applications, J. Mater. Chem. A, 2017, 5, 1446314479; (c) T. Sun, J. Xie, W. Guo, D. S. Li and Q. Zhang, Covalent-Organic Frameworks: Advanced Organic Electrode Materials for Rechargeable Batteries, $A d v$. Energy Mater., 2020, 10, 1904199; (d) Y. Zhi, Z. Wang, H.-L. Zhang and Q. Zhang, Recent Progress in Metal-Free Covalent Organic Frameworks as Heterogeneous catalysts, Small, 2020, 16, 2001070.

10 (a) M. Sommer, Conjugated Polymers Based on Naphthalene Diimide for Organic Electronics, J. Mater.
Chem. C, 2014, 2, 3088-3098; (b) S. Nam, S.-G. Hahm, D. Khim, H. Kim, T. Sajoto, M. Ree, S.-R. Marder, T.-D. Anthopoulos, D.-D.-C. Bradley and Y. Kim, Pronounced Side Chain Effects in Triple Bond-Conjugated Polymers Containing Naphthalene Diimides for n-Channel Organic Field-Effect Transistors, ACS Appl. Mater. Interfaces, 2018, 10, 12921-12929; (c) X. Guo and M. D. Watson, Conjugated Polymers from Naphthalene Bisimide, Org. Lett., 2008, 10, 5333-5336.

11 (a) W. Chen, M. Nakano, K. Takimiya and Q. Zhang, Selective Thionation of Naphtho [2,3-b] Thiophene Diimide: Tuning of the Optoelectronic Properties and Packing Structure, Org. Chem. Front., 2017, 4, 704-710; (b) W. Qiu, S. Chen, X. Sun, Y. Liu and D. Zhu, Suzuki Coupling Reaction of 1, 6, 7, 12-Tetrabromoperylene Bsimide, Org. Lett., 2006, 8, 867-870.

12 T. Maeda, J. Zhou, Y. Oda, H. Nakazumi and S. Yagi, Synthesis and Properties of Functional Dyes with Squaraine-Naphthalene Diimide Hybrid Structure, Res. Chem. Intermed., 2018, 44, 4783-4795.

13 (a) M.-A. Kobaisi, S.-V. Bhosale, K. Latham, A. M. Raynor and S.-V. Bhosale, Functional Naphthalene Diimides: Synthesis, Properties, and Applications, Chem. Rev., 2016, 116, 11685-11796; (b) A. Nowak-Krol, K. Shoyama, M. Stolte and F. Würthner, Naphthalene and Perylene Diimides-Better Alternatives to Fullerenes for Organic Electronics?, Chem. Commun., 2018, 54, 13763-13772; (c) N. Zhou and A. Facchetti, Naphthalenediimide (NDI) Polymers for All-Polymer Photovoltaics, Mater. Today, 2018, 21, 377-390; (d) W. Chen, M. Nakano, J.-H. Kim, K. Takimiya and Q. Zhang, Naphtho [2, 3-b] Thiophene Diimide (NTI): A Mono-Functionalisable Core-Extended Naphthalene Diimide for Electron-Deficient Architectures, J. Mater. Chem. C, 2016, 4, 8879-8883.

14 X. Gao, W. Qiu, X. Yang, Y. Liu, Y. Wang, H. Zhang, T. Qi, Y. Liu, K. Lu, C. Du, Z. Shuai, G. Yu and D. Zhu, First Synthesis of 2,3,6,7-Tetrabromonaphthalene Diimide, Org. Lett., 2007, 9, 3917-3920.

15 C. Röger and F. Würthner, Core-Tetrasubstituted Naphthalene Diimides: Synthesis, Optical Properties, and Redox Characteristics, J. Org. Chem., 2007, 72, 8070-8075.

16 J.-R. Mulder, C.-F. Guerra, J.-C. Slootweg, K. Lammertsma and F.-M. Bickelhaupt, Substituent Effects on the Optical Properties of Naphthalenediimides: A Frontier Orbital Analysis across the Periodic Table, J. Comput. Chem., 2016, 37, 304-313.

17 A. Insuasty, S. Maniam and S.-J. Langford, Recent Advances in the Core-Annulation of Naphthalene Diimides, Chem. - Eur. J., 2019, 25, 7058-7073.

18 S.-L. Suraru and F. Würthner, Strategies for the Synthesis of Functional Naphthalene Diimides, Angew. Chem., Int. Ed., 2014, 53, 7428-7448.

19 A.-A. Berezin, A. Sciutto, N. Demitri and D. Bonifazi, Rational Synthesis of AB-Type N-Substituted CoreFunctionalized Naphthalene Diimides (cNDIs), Org. Lett., 2015, 17, 1870-1873. 
20 D. Polpanich, U. Asawapirom, R. Thiramanas and P. Piyakulawat, Self-Colored Nanoparticles Containing Naphthalene-Bisimide Derivatives: Synthesis and Protein Adsorption Study, Mater. Chem. Phys., 2011, 129, 495-500.

21 R. Steyrleuthner, R.-D. Pietro, B.-A. Collins, F. Polzer, S. Himmelberger, M. Schubert, Z. Chen, S. Zhang, A. Salleo, H. Ade, A. Facchetti and D. Neher, The Role of Regioregularity, Crystallinity, and Chain Orientation on Electron Transport in a High-Mobility n-Type Copolymer, J. Am. Chem. Soc., 2014, 136, 4245-4256.

22 M. Sasikumar, Y.-V. Suseela and T. Govindaraju, Dibromohydantoin: A Convenient Brominating Reagent for 1,4,5,8-Naphthalenetetracarboxylic Dianhydride, Asian J. Org. Chem., 2013, 2, 779-785.

23 Y.-V. Suseela, M. Sasikumar and T. Govindaraju, An Effective and Regioselective Bromination of 1,4,5,8Naphthalenetetracarboxylic Dianhydride Using Tribromoisocyanuric Acid, Tetrahedron Lett., 2013, 54, 6314-6318.

24 T.-D. Bell, S. Yap, C.-H. Jani, S.-V. Bhosale, J. Hofkens, F.-C. De Schryver, S.-J. Langford and K.-P. Ghiggino, Synthesis and Photophysics of Core-Substituted Naphthalene Diimides: Fluorophores for Single Molecule Applications, Chem. - Asian J., 2009, 4, 1542-1550.

25 K. Xiong and Y. Xiao, Synthesis of Tetraalkyl Naphthalene Bisanhydride and Its Model Condensations with Amines, Tetrahedron Lett., 2013, 54, 3171-3175.

26 M.-M. Alam and S.-A. Jenekhe, Efficient Solar Cells from Layered Nanostructures of Donor and Acceptor Conjugated Polymers, Chem. Mater., 2004, 16, 4647-4656.

27 J. Zhao, G. Li, C. Wang, W. Chen, S.-C.-J. Loo and Q. Zhang, A New N-Substituted Heteroacene Can Detect $\mathrm{CN}^{-}$and $\mathrm{F}^{-}$Anions via Anion- $\pi$ Interaction, $R S C A d v$., 2013, 3, 9653-9657.

28 J. Zhao, J.-I. Wong, J. Gao, G. Li, G. Xing, H. Zhang, T. C. Sum, H.-Y. Yang, Y. Zhao, S.-L. Ake Kjelleberg, W. Huang, S.-C.-J. Loo and Q. Zhang, Larger $\pi$-Extended Anti-/Syn-Aroylenediimidazole Polyaromatic Compounds: Synthesis, Physical Properties, Self-Assembly, and QuasiLinear Conjugation Effect, RSC Adv., 2014, 4, 1782217831.

29 Y. Ma, X. Zhang, S. Stappert, Z. Yuan, C. Li and K. Mullen, (o-Phenyleno) Naphthalene Diimides: A Pink Fluorescent Chromophore, Chem. Commun., 2017, 53, 5310-5313.

30 H. Krüger, S. Janietz, D. Sainova, D. Dobreva, N. Koch and A. Vollmer, Hybrid Supramolecular Naphthalene DiimideThiophene Structures and Their Application in Polymer Electronics, Adv. Funct. Mater., 2007, 17, 37153723.

31 Y. Hu, Z. Wang, X. Yang, Z. Zhao, W. Han, W. Yuan, H. Li, X. Gao and D. Zhu, A Facile Synthesis of 2, 3, 6, 7-Tetrabromonaphthalene Diimides toward New $\pi$-Extended Naphthalene Diimides, Tetrahedron Lett., 2013, 54, 2271-2273.

32 F. Doria, M.-D. Antonio, M. Benotti, D. Verga and M. Freccero, Substituted Heterocyclic Naphthalene
Diimides with Unexpected Acidity. Synthesis, Properties, and Reactivity, J. Org. Chem., 2009, 74, 86168625.

33 J. Gao, Y. Li and Z. Wang, Synthesis and Properties of Naphthobisbenzothiophene Diimides, Org. Lett., 2013, 15, 1366-1369.

34 Y. Fukutomi, M. Nakano, J.-Y. Hu, I. Osaka and K. Takimiya, Naphthodithiophenediimide (NDTI): Synthesis, Structure, and Applications, J. Am. Chem. Soc., 2013, 135, 11445-11448.

35 X. Guo, F.-S. Kim, M.-J. Seger, S.-A. Jenekhe and M.-D. Watson, Naphthalene Diimide-Based Polymer Semiconductors: Synthesis, Structure-Property Correlations, and n-Channel and Ambipolar Field-Effect Transistors, Chem. Mater., 2012, 24, 1434-1442.

36 M.-J. Sung, M. Huang, S. H. Moon, T.-H. Lee, S.-Y. Park, J.-Y. Kim, S.-K. Kwon, H. Choi and Y.-H. Kim, Naphthalene Diimide-Based Small Molecule Acceptors for Fullerene-Free Organic Solar Cells, Sol. Energy, 2017, 150, 90-95.

37 H.-F. Higginbotham, P. Pander, R. Rybakiewicz, M.-K. Etherington, S. Maniam, M. Zagorska, A. Pron and A.-P. Monkman, Triphenylamine Disubstituted Naphthalene Diimide: Elucidation of Excited States Involved in TADF and Application in Near-Infrared Organic Light Emitting Diodes, J. Mater. Chem. C, 2018, 6, 8219-8225.

38 Z.-C. Chen, R.-R. Fang, Y.-Y. Yu, J.-H. Gao and J.-H. Wan, Diketopyrrolopyrrole Tailoring Charge Transport Characteristics of Naphthalene Diimide Based Polymers: From Unipolar n-Typed to Ambipolar Polymers, J. Appl. Polym. Sci., 2019, 136, 46926.

39 (a) B. Leng, D. Lu, X. Jia, X. Yang and X. Gao, Synthesis of Monolateral and Bilateral Sulfur-Heterocycle Fused Naphthalene Diimides (NDIs) from Monobromo and Dibromo NDIs, Org. Chem. Front., 2015, 2, 372-377; (b) D. Lu, X.-C. Yang, B. Leng, X.-D. Yang, C.-W. Ge, X.-S. Jia and X.-K. Gao, Fine-Tuning the Molecular Energy Levels by Incorporating Thiophene Units onto the $\pi$-Backbone of Core-Expanded Naphthalene Diimides, Chin. Chem. Lett., 2016, 27, 1022-1026.

40 (a) Y. Hu, Z. Wang, X. Zhang, X. Yang, C. Ge, L. Fu and $\mathrm{X}$. Gao, A Class of Electron-Transporting Vinylogous Tetrathiafulvalenes Constructed by the Dimerization of Core-Expanded Naphthalenediimides, Org. Lett., 2017, 19, 468-471; (b) W. Wu, J. Li, Z. Zhao, X. Yang and X. Gao, Synthesis of Largely $\pi$-Extended Naphthalenediimides via C-H Activation towards Highly Soluble and Narrow Bandgap Organic Optoelectronic Materials, Org. Chem. Front., 2017, 4, 823-827.

41 X. Gao, C. Di, Y. Hu, X. Yang, H. Fan, F. Zhang, Y. Liu, H. Li and D. Zhu, Core-Expanded Naphthalene Diimides Fused with 2-(1, 3-Dithiol-2-ylidene) Malonitrile Groups for High-Performance, Ambient-Stable, SolutionProcessed n-Channel Organic Thin Film Transistors, J. Am. Chem. Soc., 2010, 132, 3697-3699. 
42 Y. Hu, Y. Qin, X. Gao, F. Zhang, C. Di, Z. Zhao, H. Li and D. Zhu, One-Pot Synthesis of Core-Expanded Naphthalene Diimides: Enabling N-Substituent Modulation for Diverse n-Type Organic Materials, Org. Lett., 2012, 14, 292-295.

43 Z. Zhao, F. Zhang, Y. Hu, Z. Wang, B. Leng, X. Gao, C. Di and D. Zhu, Naphthalenediimides Fused with 2-(1, 3-Dithiol-2-ylidene) Acetonitrile: Strong Electron-Deficient Building Blocks for High-Performance n-Type Polymeric Semiconductors, ACS Macro Lett., 2014, 3, 1174-1177.

44 Y. Hu, Z. Wang, X. Zhang, X. Yang, H. Li and X. Gao, New Core-Expanded Naphthalene Diimides for n-Channel Organic Thin Film Transistors, Chin. J. Chem., 2013, 31, 1428-1438.

45 Y. Hu, X. Gao, C. A. Di, X. Yang, F. Zhang, Y. Liu, H. Li and D. Zhu, Core-Expanded Naphthalene Diimides Fused with Sulfur Heterocycles and End-Capped with ElectronWithdrawing Groups for Air-Stable Solution-Processed n-Channel Organic Thin Film Transistors, Chem. Mater., 2011, 23, 1204-1215.

46 H. Luo, Z. Cai, L. Tan, Y. Guo, G. Yang, Z. Liu, G. Zhang, D. Zhang, W. Xu and Y. Liu, Solution-Processed CoreExtended Naphthalene Diimides toward Organic n-Type and Ambipolar Semiconductors, J. Mater. Chem. C, 2013, 1, 2688-2695.

47 L. Tan, Y. Guo, G. Zhang, Y. Yang, D. Zhang, G. Yu, W. Xu and Y. Liu, New Air-Stable Solution-Processed Organic n-Type Semiconductors Based on Sulfur-Rich CoreExpanded Naphthalene Diimides, J. Mater. Chem. C, 2011, 21, 18042-18048.

48 L. Tan, Y. Guo, Y. Yang, G. Zhang, D. Zhang, G. Yu, W. Xu and Y. Liu, New Tetrathiafulvalene Fused-Naphthalene Diimides for Solution-Processible and Air-Stable p-Type and Ambipolar Organic Semiconductors, Chem. Sci., 2012, 3, 2530-2541.

49 W. Wu, Z. Zhao, J. Li, M. Chen and X. Gao, New $\pi$-Extended Naphthalene Diimides for High-Performance n-Type Organic Semiconductors with NIR Absorption Properties, Asian J. Org. Chem., 2018, 7, 2279-2284.

50 S.-V. Bhosale, C. Jani, C.-H. Lalander and S.-J. Langford, Solvophobic Control of Core-Substituted Naphthalene Diimide Nanostructures, Chem. Commun., 2010, 46, 973975.

51 S.-R. Bobe, S.-V. Bhosale, L. Jones, A.-L. Puyad, A.-M. Raynor and S.-V. Bhosale, A Near-Infrared Fluoride Sensor Based on A Substituted NaphthalenediimideAnthraquinone Conjugate, Tetrahedron Lett., 2015, 56, 4762-4766.

52 S.-V. Bhosale, S.-V. Bhosale, M.-B. Kalyankar and S.-J. Langford, A Core-Substituted Naphthalene Diimide Fluoride Sensor, Org. Lett., 2009, 11, 5418-5421.

53 Q. Ye, J. Chang, K.-W. Huang, X. Shi, J. Wu and C. Chi, Cyanated Diazatetracene Diimides with Ultrahigh Electron Affinity for n-Channel Field Effect Transistors, Org. Lett., 2013, 15, 1194-1197.

54 K. Cai, Q. Yan and D. Zhao, Large Hydroazaacene Diimides: Synthesis, Tautomerism, Halochromism, and
Redox-Switchable NIR Optics, Chem. Sci., 2012, 3, 31753182.

55 B. L. Hu, K. Zhang, C. An, W. Pisula and M. Baumgarten, Thiadiazoloquinoxaline-Fused Naphthalenediimides for n-Type Organic Field-Effect Transistors (OFETs), Org. Lett., 2017, 19, 6300-6303.

56 N. Banerji, S.-V. Bhosale, I. Petkova, S.-J. Langford and E. Vauthey, Ultrafast Excited-State Dynamics of Strongly Coupled Porphyrin/Core-Substituted-Naphthalenediimide Dyads, Phys. Chem. Chem. Phys., 2011, 13, 1019-1029.

57 K. Cai, J. Xie, X. Yanga and D. Zhao, Heterohexacene Diimides: Anti- and Syn-Isomers and Quinonoid Forms, Org. Lett., 2014, 16, 1852-1855.

58 X. Chen, Y. Guo, L. Tan, G. Yang, Y. Li, G. Zhang, Z. Liu, W. $\mathrm{Xu}$ and D. Zhang, Dithiazole-Fused Naphthalene Diimides toward New n-Type Semiconductors, J. Mater. Chem. C, 2013, 1, 1087-1092.

59 X. Chen, J. Wang, G. Zhang, Z. Liu, W. Xu and D. Zhang, New Core-Expanded Naphthalene Diimides with Different Functional Groups for Air-Stable Solution-Processed Organic n-Type Semiconductors, New J. Chem., 2013, 37, 1720-1727.

60 S. Maniam, H.-F. Higginbotham, S.-X. Guo, T.-D. M. Bell, E.-I. Izgorodina and S.-J. Langford, A Redox Switchable Dihydrobenzo[b]pyrazine Push-Pull System, Asian J. Org. Chem., 2014, 3, 619-623.

61 C. Li, C. Xiao, Y. Li and Z. Wang, Synthesis and Properties of Heterocyclic Acene Diimides, Org. Lett., 2013, 15, 682685.

62 W. Yue, J. Gao, Y. Li, W. Jiang, S. Di Motta, F. Negri and Z. Wang, One-Pot Synthesis of Stable NIR Tetracene Diimides via Double Cross-Coupling, J. Am. Chem. Soc., 2011, 133, 18054-18057.

63 X. Cui, C. Xiao, T. Winands, T. Koch, Y. Li, L. Zhang, N.-L. Doltsinis and Z. Wang, Hexacene Diimides, J. Am. Chem. Soc., 2018, 140, 12175-12180.

64 K. Cai, J. Xie and D. Zhao, NIR J-aggregates of Hydroazaheptacene Tetraimides, J. Am. Chem. Soc., 2014, 136, 28-31.

65 H. Luo, D. He, Y. Zhang, S. Wang, H. Gao, J. Yan, Y. Cao, Z. Cai, L. Tan, S. Wu, L. Wang and Z. Liu, Synthesis of Heterocyclic Core-Expanded Bis-Naphthalene Tetracarboxylic Diimides, Org. Lett., 2019, 21, 97349737.

66 J. Xie, K. Shi, K. Cai, D. Zhang, J. Y. Wang, J. Pei and D. Zhao, A NIR Dye with High-Performance n-Type Semiconducting Properties, Chem. Sci., 2016, 7, 499-504.

67 S. Guo, W. Wu, H. Guo and J. Zhao, Room-Temperature Long-Lived Triplet Excited States of Naphthalenediimides and Their Applications as Organic Triplet Photosensitizers for Photooxidation and Triplet-Triplet Annihilation Upconversions, J. Org. Chem., 2012, 77, 3933-3943.

68 C. Röger and F. Würthner, Core-Tetrasubstituted Naphthalene Diimides: Synthesis, Optical Properties, and Redox Characteristics, J. Org. Chem., 2007, 72, 8070-8075. 
69 J. Míšek, A.-V. Jentzsch, S.-I. Sakurai, D. Emery, J. Mareda and S. Matile, A Chiral and Colorful Redox Switch: Enhanced $\pi$ Acidity in Action, Angew. Chem., Int. Ed., 2010, 49, 7680-7683.

70 S.-L. Suraru and F. Würthner, Regioselectivity in Sequential Nucleophilic Substitution of Tetrabromonaphthalene Diimides, J. Org. Chem., 2013, 78, 5227-5238.

71 S.-L. Suraru, C. Burschka and F. Würthner, DiindoleAnnulated Naphthalene Diimides: Synthesis and Optical and Electronic Properties of Syn- and Anti-Isomers, J. Org. Chem., 2014, 79, 128-139.

72 Q. Ye, J. Chang, K.-W. Huang and C. Chi, ThiopheneFused Tetracene Diimide with Low Band Gap and Ambipolar Behavior, Org. Lett., 2011, 13, 5960-5963.

73 F. Würthner and S.-L. Suraru, Core-Tetrasubstituted Naphthalene Diimides by Stille Cross-Coupling Reactions and Characterization of Their Optical and Redox Properties, Synthesis, 2009, 1841-1845.

74 P.-Y. Gu, Z. Wang, G. Liu, L. Nie, R. Ganguly, Y. Li and Q. Zhang, Synthesis, Physical Properties, and Sensing Behaviour of a Novel Naphthalenediimide Derivative, Dyes Pigm., 2016, 131, 224-230.

75 X. Chen, G. Zhang, H. Luo, Y. Li, Z. Liu and D. Zhang, Ambipolar Charge-Transport Property for the D-A Complex with Naphthalene Diimide Motif, J. Mater. Chem. C, 2014, 2, 2869-2876.

76 (a) S.-L. Suraru, U. Zschieschang, H. Klauk and F. Würthner, A Core-Extended Naphthalene Diimide as A p-Channel Semiconductor, Chem. Commun., 2011, 47, 11504-11506; (b) L. E. Polander, S. P. Tiwari, L. Pandey, B. M. Seifried, Q. Zhang, S. Barlow, C. Risko, J.-L. Brédas, B. Kippelen and S. R. Marder, Solution-Processed Molecular Bis(Naphthalene Diimide) Derivatives with High Electron Mobility, Chem. Mater., 2011, 23, 34083410.

77 Z. Yuan, Y. Ma, T. Gessner, M. Li, L. Chen, M. Eustachi, R. T. Weitz, C. Li and K. Mullen, Core-Fluorinated Naphthalene Diimides: Synthesis, Characterization, and Application in n-Type Organic Field-Effect Transistors, Org. Lett., 2016, 18, 456-459.

78 J. Chang, Q. Ye, K.-W. Huang, J. Zhang, Z. K. Chen, J. Wu and C. Chi, Stepwise Cyanation of Naphthalene Diimide for n-Channel Field-Effect Transistors, Org. Lett., 2012, 14, 2964-2967.

79 Y. Kumar, S. Kumar, K. Mandal and P. Mukhopadhyay, Isolation of Tetracyano-Naphthalenediimide and Its Stable PlanarRadical Anion, Angew. Chem., Int. Ed., 2018, 57, 16318-16322.

80 (a) D. Shukla, S. F. Nelson, D. C. Freeman, M. Rajeswaran, W. G. Ahearn, D. M. Meyer and J. T. Carey, Thin-Film Morphology Control in Naphthalene-Diimide-Based Semiconductors: High Mobility n-Type Semiconductor for Organic Thin-Film Transistors, Chem. Mater., 2008, 20, 7486-7491; (b) Z. Liu, G. Zhang, Z. Cai, X. Chen, H. Luo, Y. Li, J. Wang and D. Zhang, New Organic
Semiconductors with Imide/Amide-Containing Molecular Systems, Adv. Mater., 2014, 26, 6965-6977.

81 (a) L. Yang, C. Xiao, W. Jiang and Z. Wang, Conjugated Donor-Acceptor Copolymers from Dicyanated Naphthalene Diimide, Tetrahedron, 2014, 70, 6265-6270; (b) H. Usta, A. Facchetti and T.-J. Marks, n-Channel Semiconductor Materials Design for Organic Complementary Circuits, Acc. Chem. Res., 2011, 44, 501510.

82 (a) X. Gao and Y. Hu, Development of n-Type Organic Semiconductors for Thin Film Transistors: A Viewpoint of Molecular Design, J. Mater. Chem. C, 2014, 2, 3099-3117; (b) B.-A. Jones, A. Facchetti, T.-J. Marks and M.-R. Wasielewski, Cyanonaphthalene Diimide Semiconductors for Air-Stable, Flexible, and Optically Transparent n-Channel Field-Effect Transistors, Chem. Mater., 2007, 19, 2703-2705.

83 (a) J. Zhang, J. Jin, H. Xu, Q. Zhang and W. Huang, Recent Progress on Organic Donor-Acceptor Complexes as Active Elements in Organic Field-Effect Transistors, J. Mater. Chem. C, 2018, 6, 3485-3498; (b) J. Zhang, G. Liu, Y. Zhou, G. Long, P. Gu and Q. Zhang, Solvent Accommodation: Functionalities Can Be Tailored Through CoCrystallization Based on 1: 1 Coronene- $\mathrm{F}_{4}$ TCNQ ChargeTransfer Complex, ACS Appl. Mater. Interfaces, 2017, 9, 1183-1188; (c) H. Xu, Y. Zhou, J. Zhang, J. Jin, G. Liu, Y. Li, R. Ganguly, L. Huang, W. Xu, D. Zhu, W. Huang and Q. Zhang, Polymer-Assisted Single Crystal Engineering of Organic Semiconductors to Alter Electron Transport, ACS Appl. Mater. Interfaces, 2018, 10, 11837-11842; (d) J. Zhang, P. Gu, G. Long, R. Ganguly, Y. Li, N. Aratani, H. Yamada and Q. Zhang, Switching Charge-Transfer Characteristics from p-Type to n-Type Through Molecular "Doping”(Co-Crystallization), Chem. Sci., 2016, 7, 38513856; (e) J. Zhang, C. Wang, G. Long, N. Aratani, H. Yamada and Q. Zhang, Fusing N-Heteroacene Analogues into One "Kinked" Molecule with Slipped TwoDimensional Ladder-like Packing, Chem. Sci., 2016, 7, 1309-1313; $(f)$ W. Chen, J. Zhang, G. Long, Y. Liu and Q. Zhang, From Non-Detectable to Decent: Replacement of Oxygen with Sulfur in Naphthalene Diimide Boosts Electron Transport in Organic Thin-Film Transistors (OTFT), J. Mater. Chem. C, 2015, 3, 8219-8224; $(g)$ J. Li, J. Miao, G. Long, J. Zhang, Y. Li, R. Ganguly, Y. Zhao, Y. Liu, B. Liu and Q. Zhang, N-Heteroheptacenequinone and N-Heterononacenequinone: Synthesis, Physical Properties, Crystal Structures and Photoelectrochemical Behaviors, J. Mater. Chem. C, 2015, 3, 9877-9884; (h) P. Gu, J. Zhang, G. Long, Z. Wang and Q. Zhang, Solution-Processable Thiadiazoloquinoxaline-Based Donor-Acceptor Small Molecules for Thin-Film Transistors, J. Mater. Chem. C, 2016, 4, 3809-3814.

84 (a) T. He, M. Stolte and F. Würthner, Air-Stable n-Channel Organic Single Crystal Field-Effect Transistors Based on Microribbons of Core-Chlorinated Naphthalene Diimide, Adv. Mater., 2013, 25, 6951-6955; (b) J.-H. Oh, S.-L. Suraru, 
W.-Y. Lee, M. Könemann, H. W. Höffken, C. Röger, R. Schmidt, Y. Chung, W. C. Chen, F. Würthner and Z. Bao, High-Performance Air-Stable n-Type Organic Transistors Based on Core-Chlorinated Naphthalene Tetracarboxylic Diimides, Adv. Funct. Mater., 2010, 20, 2148-2156.

85 X. Cui, C. Xiao, L. Zhang, Y. Li and Z. Wang, Polycyclic Aromatic Hydrocarbons with Orthogonal Tetraimides as n-Type Semiconductors, Chem. Commun., 2016, 52, 13209-13212.

86 K. Takimiya and M. Nakano, Thiophene-Fused Naphthalene Diimides: New Building Blocks for Electron Deficient $\pi$-Functional Materials, Bull. Chem. Soc. Jpn., 2018, 91, 121-140.

87 (a) Q. Liu, S.-E. Bottle and P. Sonar, Developments of Diketopyrrolopyrrole-Dye-Based Organic Semiconductors for A Wide Range of Applications in Electronics, $A d v$. Mater., 2020, 32, 1903882.

88 F. Zhang, Y. Hu, T. Schuettfort, C.-A. Di, X. Gao, C.-R. McNeill, L. Thomsen, S.-C. Mannsfeld, W. Yuan, H. Sirringhaus and D. Zhu, Critical Role of Alkyl Chain Branching of Organic Semiconductors in Enabling Solution-Processed n-Channel Organic Thin-Film Transistors with Mobility of up to $3.50 \mathrm{~cm}^{2} \mathrm{~V}^{-1} \mathrm{~s}^{-1}, J$. Am. Chem. Soc., 2013, 135, 2338-2349.

89 F. Zhang, C.-A. Di, N. Berdunov, Y. Hu, Y. Hu, X. Gao, Q. Meng, H. Sirringhaus and D. Zhu, Ultrathin Film Organic Transistors: Precise Control of Semiconductor Thickness via Spin-Coating, Adv. Mater., 2013, 25, 14011407.

90 K. Wu, H. Li, L. Li, S. Zhang, X. Chen, Z. Xu, X. Zhang, W. Hu, L. Chi, X. Gao and Y. Meng, Controlled Growth of Ultrathin Film of Organic Semiconductors by Balancing the Competitive Processes in Dip-Coating for Organic Transistors, Langmuir, 2016, 32, 6246-6254.

91 (a) J. Locklin, M. E. Roberts, S. C. B. Mannsfeld and Z. Bao, Optimizing the Thin Film Morphology of Organic Field-Effect Transistors: The Influence of Molecular Structure and Vacuum Deposition Parameters on Device Performance, J. Macromol. Sci., Polym. Rev., 2006, 46, 79101; (b) J. Sun, R. Devine, B. M. Dhar, B. J. Jung, K. C. See and H. E. Katz, Improved Morphology and Performance from Surface Treatments of Naphthalenetetracarboxylic Diimide Bottom Contact Field-Effect Transistors, ACS Appl. Mater. Interfaces, 2009, 1, 1763-1769; (c) G. Generali, F. Dinelli, R. Capelli, S. Toffanin, F. di Maria, M. Gazzano, G. Barbarella and M. Muccini, Correlation among Morphology, Crystallinity, and Charge Mobility in OFETs Made of Quaterthiophene Alkyl Derivatives on a Transparent Substrate Platform, J. Phys. Chem. C, 2011, 115, 23164-23169.

92 (a) J.-L. Sessler, S. Camiolo and P.-A. Gale, Pyrrolic and Polypyrrolic Anion Binding Agents, Coord. Chem. Rev., 2003, 240, 17-55; (b) P.-A. Gale, E.-N.-W. Howe, X. Wu and M.-J. Spooner, Anion Receptor Chemistry: Highlights from 2016, Coord. Chem. Rev., 2018, 375, 333-372.
93 (a) B. Gu and Q. Zhang, Recent Advances on Functionalized Upconversion Nanoparticles for Detection of Small Molecules and Ions in Biosystems, Adv. Sci., 2018, 5, 1700609; (b) P. Gu, Z. Wang and Q. Zhang, Azaacenes as Active Elements for Sensing and Bio Applications, J. Mater. Chem. B, 2016, 4, 70607074.

94 (a) D. Buckland, S.-V. Bhosale and S.-J. Langford, A Chemodosimer Based on A Core-Substituted Naphthalene Diimide for Fluoride Ion Detection, Tetrahedron Lett., 2011, 52, 1990-1992; (b) Q. Li, M. Peng, N. Li, J. Qin and Z. Li, New Colorimetric Chemosensor Bearing Naphthalendiimide Unit with Large Blue-Shift Absorption for Naked Eyes Detection of $\mathrm{Cu}^{2+}$ Ions, Sens. Actuators, B, 2012, 173, 580-584.

95 (a) J. Luo, S. Jiang, S. Qin, H. Wu, Y. Wang, J. Jiang and $\mathrm{X}$. Liu, Highly Sensitive and Selective Turn-on Fluorescent Chemosensor for $\mathrm{Hg}^{2+}$ in Pure Water Based on A Rhodamine Containing Water-Soluble Copolymer, Sens. Actuators, B, 2011, 160, 1191-1197; (b) G. Li, H.-M. Duong, Z. Zhang, J. Xiao, L. Liu, Y. Zhao, H. Zhang, F. Huo, S. Li, J. Ma, F. Wudl and Q. Zhang, Approaching A Stable, Green Twisted Heteroacene Through "Clean Reaction" Strategy, Chem. Commun., 2012, 48, 5974-5976; (c) Q. Zhang, J. Xiao, Z.-Y. Yin, H. M. Duong, F. Qiao, F. Boey, X. Hu, H. Zhang and F. Wudl, Synthesis, Characterization, and Physical Properties of A Conjugated Heteroacene: 2-Methyl-1,4,6,7,8,- hexaphenylbenz(g)isoquinolin-3(2H)one (BIQ), Chem. - Asian J., 2011, 6, 856-862; (d) G. Li, Y. Wu, J. Gao, J. Li, Y. Zhao and Q. Zhang, Synthesis, Physical Properties, and Anion Recognition of Two Novel Larger Azaacenes: Benzannelated Hexazaheptacene and Benzannelated N, N '-Dihydrohexazaheptacene, Chem. Asian J., 2013, 8, 1574-1578.

96 (a) H. Lan, B. Liu, G. Lv, Z. Li, X. Yu, K. Liu, X. Cao, H. Yang, S. Yang and T. Yi, Dual-Channel Fluorescence "Turn on" Probe for $\mathrm{Cu}^{2+}$, Sens. Actuators, B, 2012, 173, 811-816; (b) B. Gu, M. Ye, L. Nie, Y. Fang, Z. Wang, X. Zhang, H. Zhang, Y. Zhou and Q. Zhang, Organic-DyeModified Upconversion Nanoparticle as A Multichannel Probe to Detect $\mathrm{Cu}^{2+}$ in Living Cells, ACS Appl. Mater. Interfaces, 2018, 10, 1028-1032; (c) B. Gu, Y. Zhou, X. Zhang, X. Liu, Y. Zhang, R. Marks, H. Zhang, X. Liu and Q. Zhang, Thiazole Derivative-Modified Upconversion Nanoparticles for $\mathrm{Hg}^{2+}$ Detection in Living Cells, Nanoscale, 2016, 8, 276-282; (d) Y. Zhou, W. Pei, C. Wang, J. Zhu, J. Wu, Q. Yan, L. Huang, W. Huang, C. Yao, J.-S.C. Loo and Q. Zhang, Rhodamine-Modified Upconversion Nanophosphors for Ratiometric Detection of Hypochlorous Acid in Aqueous Solution and Living cells, Small, 2014, 10, 3560-3567; (e) Y. Zhou, W. Chen, J. Zhu, W. Pei, C. Wang, L. Huang, C. Yao, Q. Yan, W. Huang, J.-S.-C. Loo and Q. Zhang, Nanoprobes: Inorganic-Organic Hybrid Nanoprobe for NIR-Excited Imaging of Hydrogen Sulfide in Cell Cultures and Inflammation in A Mouse Model, Small, 2014, 10, 4802. 
97 C. Zhou, Y. Li, Y. Zhao, J. Zhang, W. Yang and Y. Li, An Unusual Addition Reaction for Constructing A Novel pH-Controlled Fluorescence Switch, Org. Lett., 2011, 13, 292-295.

98 Y. Li, C. Zhang, Z. Li, P. Gu, Z. Wang, H. Li, J. Lu and Q. Zhang, Controlled Deposition of Large-area and Highly-Ordered Thin Films: Effect of Dip-Coating-Induced Morphological Evolution on Resistive Memory Performance, J. Mater. Chem. C, 2019, 7, 3512-3521.

99 C. Zhang, H. Li, Z. Li, Y. Li, Q.-J. Zhang and J.-M. Lu, Solvent-Vapor Annealing of Amphiphile/Metal Interface for Orientated Molecular Stacking and Upgraded Resistive Memory Performance, Macromol. Chem. Phys., 2019, 220, 1900334.

100 C. Zhang, H. Li, Y. Su, Q. Zhang, Y. Li and J. Lu, Controllable and Versatile Electrophoretic Deposition Technology for Monolithic Organic Memory Devices, ACS Appl. Mater. Interfaces, 2020, 12, 15482-15490.

101 (a) J. Rivnay, S.-C. Mannsfeld, C.-E. Miller, A. Salleo and M.-F. Toney, Quantitative Determination of Organic Semiconductor Microstructure from the Molecular to Device Scale, Chem. Rev., 2012, 112, 5488-5519; (b) J. Xiao, Z. Yin, H. Li, Q. Zhang, F. Boey, H. Zhang and Q. Zhang, Postchemistry of Organic Particles: When TTF Microparticles Meet TCNQ Microstructures in Aqueous Solution, J. Am. Chem. Soc., 2010, 132, 6926-6928; (c) B. Yang, J. Xiao, J.-I. Wong, J. Guo, Y. Wu, L. Ong, L.-L. Lao, F. Boey, H. Zhang, H. Yang and Q. Zhang, Shape-Controlled Micro/Nanostructures of 9, 10-Diphenylanthracene (DPA) and Their Application in Light-Emitting Devices, J. Phys. Chem. C, 2011, 115, 79247927; (d) J. Xiao, H. Yang, Z. Yin, J. Guo, F. Boey, H. Zhang and Q. Zhang, Preparation, Characterization, and Photoswitching/Light-Emitting Behaviors of Coronene Nanowires, J. Mater. Chem., 2011, 21, 1423-1427; (e) J. Xiao, Z. Yin, Y. Wu, J. Guo, Y. Cheng, H. Li, Y.-Z. Huang, Q. Zhang, J. Ma, F. Boey, H. Zhang and Q. Zhang, Chemical Reaction Between Ag Nanoparticles and TCNQ Microparticles in Aqueous Solution, Small, 2011, 7, 1242-1246; ( $f$ ) J. Xiao, B. Yang, J.-I. Wong, Y. Liu, F. Wei, K.-J. Tan, X. Teng, Y. Wu, L. Huang, C. Kloc, F. Boey, J. Ma, H. Zhang, H. Yang and Q. Zhang, Synthesis, Characterization, Self-Assembly, and Physical Properties of 11-Methylbenzo[d]pyreno[4,5-b]furan, Org. Lett., 2011, 13, 3004-3007; (g) Z.-Q. Lin, P.-J. Sun, Y.-Y. Tay, J. Liang, Y. Liu, N.-E. Shi, L.-H. Xie, M.-D. Yi, Y. Qian, Q.-L. Fan, H. Zhang, H.-H. Hng, J. Ma, Q. Zhang and W. Huang, Kinetically Controlled Assembly of A Spirocyclic Aromatic Hydrocarbon into Polyhedral Micro/ Nanocrystals, ACS Nano, 2012, 6, 5309-5319; (h) J. Wu, Y. Sun, W.-B. Pei, L. Huang, W. Xu and Q. Zhang, Polypyrrole Nanotube Film for Flexible Thermoelectric Application, Synth. Met., 2014, 196, 173-177; (i) C.-E. Zhao, J. Wu, S. Kjelleberg, J.-S.-C. Loo and Q. Zhang, Employing A Flexible and Low-Cost Polypyrrole Nanotube Membrane as An Anode to Enhance Current
Generation in Microbial Fuel Cells, Small, 2015, 11, 34403443; (j) J. Xie, C.-E. Zhao, Z. Lin, P. Gu and Q. Zhang, Nanostructured Conjugated Polymers for Energy-Related Applications beyond Solar Cells, Chem. - Asian J., 2016, 11, 1489-1511.

102 T. Nakamura, N. Shioya, T. Shimoaka, R. Nishikubo, T. Hasegawa, A. Saeki, Y. Murata, R. Murdey and A. Wakamiya, Molecular Orientation Change in Naphthalene Diimide Thin Films Induced by Removal of Thermally Cleavable Substituents, Chem. Mater., 2019, 31, 1729-1737.

103 A.-O.-F. Jones, B. Chattopadhyay, Y.-H. Geerts and R. Resel, Substrate-Induced and Thin-Film Phases: Polymorphism of Organic Materials on Surfaces, Adv. Funct. Mater., 2016, 26, 2233-2255.

104 S. Milita, F. Liscio, L. Cowen, M. Cavallini, B.-A. Drain, T. Degousée, S. Luong, O. Fenwick, A. Guagliardi, B.-C. Schroeder and N. Masciocchi, Polymorphism in N, N'-dialkyl-Naphthalene Diimides, J. Mater. Chem. C, 2020, 8, 3097-3112.

105 S.-M. Wagalgave, D. DucLa, R.-S. Bhosale, M. A. Kobaisi, L.-A. Jones, S.-V. Bhosale and S.-V. Bhosale, Fabrication of Diverse Nano-Architectures Through the Self-Assembly of A Naphthalene Diimide Derivative Bearing Four Carbamates, New J. Chem., 2018, 42, 6785-6793.

106 M. Diebold, E. Christ, L. Biniek, L. Karmazin, B. Heinrich, C. Contal, S. Ghosh, P.-J. Mesini and M. Brinkmann, Original Polymorphism in A Naphthalene Bisimide $\pi$-Conjugated Organogelator: A Complex Interplay Between Hydrogen Bonding and Heterocycle $\pi$-Stacking, J. Mater. Chem. C, 2019, 7, 13120-13129.

107 C. Röger, Y. Miloslavina, D. Brunner, A.-R. Holzwarth and F. Würthner, Self-Assembled Zinc Chlorin Rod Antennae Powered by Peripheral Light-Harvesting Chromophores, J. Am. Chem. Soc., 2008, 130, 5929-5939.

108 A. Sarkar, S. Dhiman, A. Chalishazar and S.-J. George, Visualization of Stereoselective Supramolecular Polymers by Chirality-Controlled Energy Transfer, Angew. Chem., Int. Ed., 2017, 56, 13767-13771.

109 S.-V. Bhosale, N.-V. Ghule, M.-A. Kobaisi, M.-M. Kelsona and S.-V. Bhosale, Controlled Nanodimensional Supramolecular Self-Assembly of Tetra-alkylated Naphthalene Diimide Derivatives, Chem. - Eur. J., 2014, 20, 10775-10781.

110 S.-V. Bhosale, M.-A. Kobaisi, R.-S. Bhosale and S.-V. Bhosale, Well-Organized Supramolecular SelfAssembly of Acene Diimide Derivatives, RSC Adv., 2013, 3, 19840-19843.

111 S.-V. Bhosale, C.-H. Jani, C.-H. Lalander, S.-J. Langford, I. Nerush, J.-G. Shapter, D. Villamain and E. Vauthey, Supramolecular Construction of Vesicles Based on CoreSubstituted Naphthalene Diimide Appended with Triethyleneglycol Motifs, Chem. Commun., 2011, 47, 82268228.

112 Y.-C. Wu, P. Leowanawat, H.-J. Sun, B.-E. Partridge, M. Peterca, R. Graf, H.-W. Spiess, X. Zeng, G. Ungar, 
C.-S. Hsu, P.-A. Heiney and V. Percec, Complex Columnar Hexagonal Polymorphism in Supramolecular Assemblies of A Semifluorinated Electron-Accepting Naphthalene Bisimide, J. Am. Chem. Soc., 2015, 137, 807819.

113 Y. Zang, F. Zhang, D. Huang, C.-A. Di, Q. Meng, X. Gao and D. Zhu, Specific and Reproducible Gas Sensors Utilizing Gas-Phase Chemical Reaction on Organic Transistors, Adv. Mater., 2014, 26, 2862-2867.

114 S.-B. Schmidt, T. Biskup, X. Jiao, C.-R. McNeill and M. Sommer, Controlling Intermolecular Redox-Doping of Naphthalene Diimides, J. Mater. Chem. C, 2019, 7, 44664474.

115 (a) B. Russ, A. Glaudell, J. J. Urban, M. L. Chabinyc and R. A. Segalman, Organic Thermoelectric Materials for Energy Harvesting and Temperature Control, Nat. Rev. Mater., 2016, 1, 16050; (b) Y. Du, J. Xu, B. Paul and P. Eklund, Flexible Thermoelectric Materials and Devices, Appl. Mater. Today, 2018, 12, 366-388; (c) H. Wang and C. Yu, Organic Thermoelectrics: Materials Preparation, Performance Optimization, and Device Integration, Joule, 2019, 3, 53-80.
116 (a) D. Kiefer, A. Giovannitti, H. Sun, T. Biskup, A. Hofmann, M. Koopmans, C. Cendra, S. Weber, L. J. Anton Koster, E. Olsson, J. Rivnay, S. Fabiano, I. McCulloch and C. Muller, Enhanced n-Doping Efficiency of a Naphthalenediimide-Based Copolymer through Polar Side Chains for Organic Thermoelectrics, ACS Energy Lett., 2018, 3, 278-285; (b) S. Wang, H. Sun, T. Erdmann, G. Wang, D. Fazzi, U. Lappan, Y. Puttisong, Z. Chen, M. Berggren, X. Crispin, A. Kiriy, B. Voit, T. J. Marks, S. Fabiano and A. Facchetti, A Chemically Doped Naphthalenediimide-Bithiazole Polymer for n-Type Organic Thermoelectrics, Adv. Mater., 2018, 30, 1801898.

117 (a) W. Zhao, F. Zhang, X. Dai, W. Jin, L. Xiang, J. Ding, X. Wang, Y. A.-O. Wan, H. Shen, Z. He, J. Wang, X. Gao, Y. Zou, C. A.-O. Di and D. Zhu, Enhanced Thermoelectric Performance of N-Type Organic Semiconductor via Electric Field Modulated Photo-Thermoelectric Effect, Adv. Mater., 2020, 2000273; (b) F. Zhang, Y. Zang, D. Huang, C.-A. Di, X. Gao, H. Sirringhaus and D. Zhu, Modulated Thermoelectric Properties of Organic Semiconductors Using Field-Effect Transistors, Adv. Funct. Mater., 2015, 25, 3004-3012. 